in der so wertvollen lateinischen Überlieferung des Codex Veronensis 60 (perg., s. VII) erhalten sind, Herr Professor Hans Lietzmann die große Güte gehabt, mir die photographische Wiedergabe zur Verfügung zu stellen, die er von dieser Handschrift besitzt.

\title{
II. Die Gruppierung der Handschriften
}

Unter den aufgezählten Handschriften gibt es einige, die ihre Bedeutung und Stellung in den Gruppen im Laufe des Werkes wechseln. Diese Abweichungen habe ich mich bemüht unmittelbar anschaulich zu machen dadurch, daß ich entsprechend die Reihenfolge änderte, in welcher die Handschriften über dem kritischen Apparat angeordnet werden.

Nach dem Wert und nach der Verwandtschaft der Handschriften lassen sich der Reihe nach die folgenden Gruppen feststellen. - Kleine Buchstaben dienen zur Bezeichnung der Handschriftengruppen. Zu erinnern ist daran, daß $B$ für fast das ganze erste Buch fehlt und an andern Stellen häufig durch Hände von verschiedenem Wert, $\mathrm{B}^{2}, \mathrm{~B}^{3}$ und $B^{4}$, ergänzt worden ist.

Erstes Buch: B A HN(n) GS(s) DL $+\mathrm{FV}(\mathrm{v})=\mathrm{z}$.

Zweites Buch: B A HN(n) + GS (s) $=$ r $\mathrm{B}^{2}\left(\right.$ oder $\left.\mathrm{B}^{3}\right) \mathrm{L}+\mathrm{FVR}(\mathrm{v})$ $=$ z. Für diese beiden Bücher stellt sich $A^{c}$, wie wir sehen werden, immer neben $\mathrm{L}$.

Drittes Buch bis S. 187: B HN (n) + GS (s) $=\mathrm{r} \quad \mathrm{AL}(\mathrm{y}) \quad \mathrm{FVR}(\mathrm{v})$.

Von S. 187 ab: B V ${ }^{2} \mathrm{HN}(\mathrm{n})+\mathrm{GSP}(\mathrm{s})=\mathrm{r} \quad \mathrm{AL}(\mathrm{y}) \mathrm{F}$. $\mathrm{AL}(\mathrm{y})$.

Von S. 249 ab: B (oder B ( $^{4} \mathrm{~V}^{2}(\operatorname{oder} \mathrm{V}) \mathrm{F} \mathrm{HN}(\mathrm{n})+\mathrm{GSP}(\mathrm{s})=\mathrm{r}$

Nach dieser Einteilung sind die besten Handschriften: für die beiden ersten Bücher $\mathrm{A}$ und $\mathrm{B}, \mathrm{zu}$ denen im ersten Buch hinzukommen $\mathrm{HN}(\mathrm{n})$; für die folgenden $B u ̈ c h e r ~ B$ und von $S .187$ ab $B\left(B^{4}\right) V^{2}(V)$, zu denen von S. 249 ab F hinzukommt. Die minderwertige Überlieferung wird von den Gruppen dargestellt, die ich der Reihe nach mit z (s stellt sich im ersten Buch sehr häufig zu z), mit $\mathrm{yv}, \mathrm{yF}$ und $\mathrm{y}$ bezeichnet habe. Die Gruppe $\mathrm{r}$ (HNGSP) endlich nimmt eine mittlere Stelle ein.

Wie ich weiter unten (S. XLVIIlff) nachweise, liefern die lateinische Ubersetzung des Cassiodor und in geringerm Grade die Abschrift T der Tripartita des Theodorus Lector ein hervorragendes Mittel zur Kontrolle. Deßhalb werde ich auch, um den Wert der Lesarten festzustellen, von jetzt ab häufig ihr Zeugnis anzurufen haben. Anderseits besitzen wir besonders für die Urkunden eine reiche Parallelüberlieferung bei Eusebius, Athanasius, Socrates, Sozomenus, Gelasius Cyzicenus, in 
den lateinischen Fassungen usw. Diese Überlieferung bildet ein ausgezeichnetes Kriterium bei Beurteilung der Lesarten. Man kann sich darauf um so mehr verlassen, als weiter unten, wie ich hoffe, der Nachweis erbracht werden wird, daß in den Handschriften Theodorets keine Spur einer Nachvergleichung oder Correctur auf Grund dieser Parallelüberlieferung vorhanden ist.

Da B erst vom dritten Buch ab fortlaufend eintritt, stelle ich seine Untersuchung vorlăufig zurück und beginne mit der Prüfung einer auf die Handschrift A bezüglichen speciellen Frage. Für die beiden ersten Bücher hat in der Tat das Zeugnis von A die größte Wichtigkeit, und man muß klar herausschälen, welches seine wirkliche Überlieferung ist, und diese von der durch Correctur zu $\mathrm{A}^{\mathrm{c}}$ umgestalteten unterscheiden.

\section{1. $A$ und $A^{c}$}

Die besondere Schwierigkeit, welche die Handschrift A bietet, besteht in folgendem. Während der ganzen ersten Hälfte d.h. etwa für die beiden ersten Bücher - eine absolut genaue Abgrenzung ist nicht möglich -, war der Schreiber einer Handschrift gefolgt, die eine im allgemeinen vortreffliche Überlieferung darstellte. Vom dritten Buch ab hat er diese Überlieferung nicht mehr gekannt oder er hat sie aufgegeben, um ausschließlich einer Handschrift zu folgen, die ein vollkommener Doppelgänger von L ist. Anderseits hat ein Schreiber den ganzen ersten Teil der Handschrift sorgfältig nach dieser zweiten Vorlage collationiert und hat sich mit leidenschaftlichem Eifer darauf geworfen, die Lesarten der ersten Handschrift zu tilgen, auszuradieren oder durchzustreichen, um die der mit $\mathrm{L}$ parallelgehenden Überlieferung an ihre Stelle zu setzen.

Ist nun die Persönlichkeit, welche die Correcturen vorgenommen hat, 'der erste Schreiber selbst, der auf solche Art seinen Text hätte einheitlich gestalten wollen? Die Photographie der Handschrift ließ mich im allgemeinen keinen entscheidenden Unterschied in der Schrift wahrnehmen. Ich habe aber das große Glück gehabt, über diesen Punkt die Meinung eines Gelehrten einholen zu können, dessen Kompetenz unbestritten ist. Thomas W. Allen ist so freundlich gewesen, für mich in der Handschrift selbst das lange Verzeichnis aller einigermaßen wichtigen Lesarten von $\mathrm{A}^{\mathrm{c}}$ zu prüfen. Nach seiner Meinung ist die Schrift des Correctors $\mathrm{A}^{\mathrm{c}}$ der von A sehr ähnlich. Der einzige greifbare Unterschied ist, daß die Tinte von $A^{\circ}$ glänzender ist als die von $A$, und dieses Kriteriums hat sich Th. W. Allen bedient, um beide zu unterscheiden. Es wäre also möglich, daß der Schreiber von $\mathrm{A}^{\mathrm{c}}$ nur der von $\mathrm{A}$ wäre, der sich selbst hinterdrein corrigierte, nachdem er sich entschieden hatte, die Handschrift aus dem Zweige L zu bevorzugen. Ich komme unten (S. XL f), 
wo ich von der Gruppe $\mathrm{AL}(\mathrm{y})$ spreche, auf die Beziehungen von A zu L zurück und auf die Erklärung, die sie zulassen. Für die Aufstellung des Textes hat die Frage, ob $\mathrm{A}^{\mathrm{c}}$ eine von $\mathrm{A}$ verschiedene Hand ist, praktisch keine große Bedeutung. Man kann obne weiteres behaupten, daß in jedem Fall die Lesarten von $A^{c}$ aus einer Handschrift geschöpft sind, die L sehr nahe steht.

Es folgt zunächst eine Reihe von Fällen, wo fehlerhafte Correcturen von $\mathrm{A}^{\mathrm{c}}$ Lesarten von $\mathrm{L}$ allein darstellen:

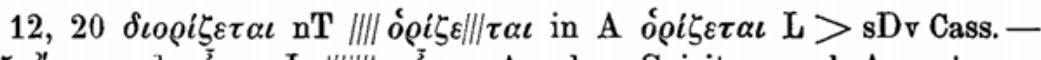
$48,5$ है $\chi o v \sigma \alpha \nu]$ ov $\sigma \alpha \nu \mathrm{L} /|I I I I|$ ov $\sigma \alpha \nu \mathrm{A}$, aber Spiritus und Accent von $\mathrm{A}^{\mathrm{c}}-51,4 \stackrel{5}{\eta} \nu^{1}=$ Athan. Socr. Gel.] $\stackrel{\circ}{\circ} \tau \iota \stackrel{3}{\eta} v \mathrm{~L} \stackrel{\circ}{\circ} \tau \iota$ übergeschrieben $\mathrm{A}^{\mathrm{c}}$

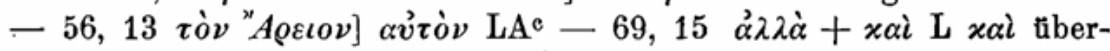
geschrieben $\mathrm{A}^{\mathrm{c}}-107,18 \pi \rho \varepsilon \sigma \beta \dot{v} \tau \varepsilon \rho o \iota+o i \mathrm{LA}^{\mathrm{c}}-114,5 \tau \iota \sigma \iota \nu-$

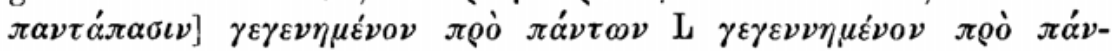

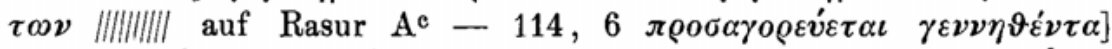

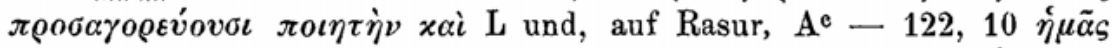
$+\chi \alpha \tau \alpha \lambda \alpha \beta \varepsilon \tilde{\imath} \nu \mathrm{L}$ und, übergeschrieben, $\mathrm{A}^{\mathrm{e}}-127,11 \dot{\varepsilon} \varphi v \gamma \dot{\alpha} \delta \varepsilon v \sigma \alpha \nu$ $=$ Athan. Socr.] $\varepsilon_{\varphi} \hat{o}^{\prime} v \varepsilon v \sigma \alpha \mathrm{L}$ und, auf Rasur, $\mathrm{A}^{\mathrm{c}}-132,16 \dot{\varepsilon} x>\mathrm{L}$, gestrichen in A.

$\mathrm{Zu}$ dieser Liste von allein in $\mathrm{A}^{\mathrm{e}} \mathrm{L}$ vorkommenden Übereinstimmungen, die ich noch verlängern könnte, sind die sehr zahlreichen Fälle hinzuzufügen, wo $\mathrm{A}^{\mathrm{c}}$ mit $\mathrm{L}+$ einer seiner $\mathrm{Z}$ willingshandschriften $\mathrm{D}, \mathrm{B}^{2}$ oder $\mathrm{B}^{3}$ zusammentrifft oder auch mit $\mathrm{L}+\mathrm{v}$ oder mit der ganzen Gruppe $\mathrm{z}$, zu der noch häufig die Gruppe s hinzukommt. Dann schöpft $\mathrm{A}^{\mathrm{c}}$ gleichfalls seine Lesarten aus einer Zwillingshandschrift von L. Einige Beispiele für solche gemeinsamen Fehler sind:

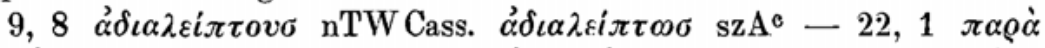

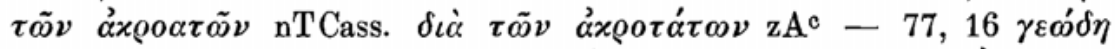

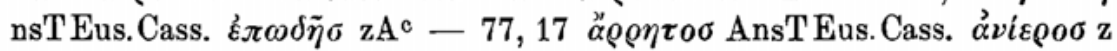
und, am Rand, $\mathrm{A}^{\mathrm{c}}-109,14$ ist die richtige Lesart $\sigma \iota \gamma \gamma \iota \delta o v ́ v o v$, die A mit

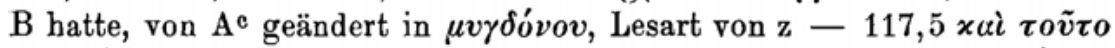

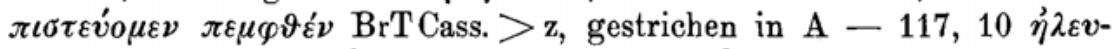

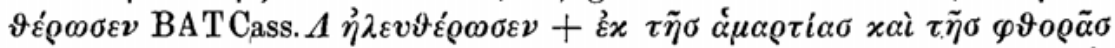
$\mathrm{rz}$ und, am Rand, $\mathrm{A}^{\mathrm{c}}-124,14 \tau \tilde{\omega} \nu^{1}>\mathrm{z}$, gestrichen in $\mathrm{A}-126,11 \pi \rho o^{-}$ $\sigma \omega \pi \alpha$ ArT Athan. Socr. $\pi \varrho o ́ \sigma \omega \pi \alpha+\tau \dot{v} \pi \tau \varepsilon \sigma \vartheta \alpha \iota x \varepsilon \lambda \varepsilon v \dot{\omega} \omega \nu \mathrm{z}$ und, am Rand, $\mathrm{A}^{\mathrm{c}}-128,16 \delta \iota x \alpha \sigma \tau \tilde{\omega} \nu+x \alpha \vartheta \dot{\varepsilon} x \alpha \sigma \tau o \nu \mathrm{Lv}$ und, am Rand, $\mathrm{A}^{\mathrm{c}}-132,16$ $\chi \alpha \tau \varepsilon \psi \eta \varphi i \sigma \alpha \nu \tau o+\alpha \dot{\alpha} \hat{o} \gamma \omega \sigma \mathrm{z}$ und, am Rand, $\mathrm{A}^{\mathrm{c}}-134,21 \varepsilon \dot{\varepsilon} \boldsymbol{\gamma}>\mathrm{Lv}, \| I I I \mid$ in $\mathrm{A}-135,20 \dot{\eta} \mu \varepsilon \rho \tilde{\omega} \nu$ ArT Cass. $\Sigma \dot{\eta} \mu \varepsilon \rho \tilde{\omega} \nu+\ddot{\eta} \mu \eta \nu \tilde{\omega} \nu \mathrm{zA}^{\mathrm{c}}-148,5$ $\sigma x \varepsilon \dot{\varepsilon} \mu \alpha \tau \sigma \varsigma] \sigma x \alpha \dot{\mu} \mu \alpha \tau \sigma \sigma \mathrm{zA}^{\mathrm{c}}$.

Analoge Beispiele kann man im kritischen Apparat fast auf jeder Seite finden. Man übersehe nicht, daß diese Correcturen, die lauter 
schlechte Lesarten einführen, ganz mechanisch und ohne jede Kritik gemacht sind. Ich will bloß noch darauf hinweisen, daß die Vorlage für $A^{c}$, obwohl sie L sehr nahe steht, nicht L selbst ist. Die Vorlage für $A^{c}$ gehört zur Gruppe $\mathbf{z}$ und steht innerhalb dieser Gruppe nur näher $\mathrm{L}$ als v. Einige fehlerhafte Correcturen von $\mathrm{A}^{\circ}$ finden sich bloß in Hand-

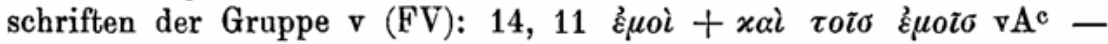

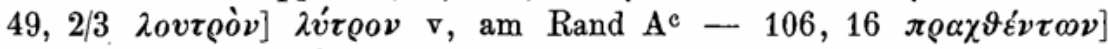
$\lambda \varepsilon \chi \vartheta \dot{v} \nu \tau \omega \nu \mathrm{F}$, am Rand $\mathrm{A}^{\mathrm{c}}$; vgl. auch die lange Variante 164, 20/21: während $\mathrm{L}$ die gute Lesart darbietet, hat $\mathrm{A}^{\mathrm{e}}$ sie getilgt, um die Variante von $\mathrm{v}$ zu übernehmen.

Weiter unten (S. XLI) wird man sehen, daß, seitdem A eine einfache Zwillingshandschrift von L geworden ist, A immer noch, wenn auch seltener, Correcturen einführt, die mit Lesarten von $\mathrm{F}$ übereinstimmen.

Besonders sorgfältig ist in dem ersten Teile des Codex A auf die Varianten zu achten, die von der ersten Niederschrift von A selbst stammen könnten, und die A aus seiner guten Quelle geschöpft hat. Auch hierbei sind mir die Mitteilungen Th. W. Allens sehr nützlich gewesen. Zum Beispiel für so wichtige Lesarten wie 5, $4 \sigma \tau \varrho \varepsilon \beta \lambda \tilde{\omega} \nu$

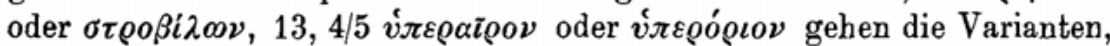
die sicher nach Th. W. Allen von der ersten Hand von A stammen, auf seine eigene Quelle zurück und nicht auf die schlechte Überlieferung von $\mathrm{A}^{\mathrm{c}}$ und gewinnen dadurch einen besondern Wert.

In der Mehrzahl der Fälle sind nach der Correctur von $\mathrm{A}^{\mathrm{c}}$ die ursprünglichen Lesarten von A nicht mehr zu lesen, aber sie lassen sich mit absoluter Sicherheit durch Vergleichung mit den besten Handschriften oder mit den Zeugnissen der Yarallelüberlieferung wiederherstellen. $\mathrm{Da} \mathrm{A}^{\mathrm{c}}$ sich immer zu einer Handschrift des Zweiges L stellt, kann der Leser tatsächlich im allgemeinen den Schluß ziehen, daß A die entgegengesetzte Lesart hatte, wie es die oben angeführten Beispiele evident beweisen.

Die besondere Verwicklung, welche die Überlieferung in A darstellt, hatte der letzte Herausgeber Gaisford nicht untersucht. Es war aber nötig die Frage aufzuklären, bevor das Zeugnis dieser wichtigen Handschrift in Anspruch genommen wird.

\section{Die Gruppe HNGSP $=\mathbf{r}$}

Indem ich nunmehr zur Prüfung der Handschriftengruppen übergehe, untersuche ich zuerst die Gruppe $r$ (HNGSP) und die beiden Einzelgruppen, aus denen sie besteht, HN(n) und GSP(s). Bei den Abweichungen der andern Handschriften läßt sich in der Tat gerade 
die Gruppe r, abgesehen von gewissen Einschränkungen hinsichtlich des ersten Buches, am gleichmäßigsten aufrechterhalten.

Es folgen Beispiele für offenkundig fehlerhafte Übereinstimmungen, welche die Gruppe HNGSP(r) vom zweiten Buch ab außer Zweifel setzen. Die verwickelteren Beziehungen von $\mathrm{HN}(\mathrm{n})$ und $\mathrm{GS}(\mathrm{s})$ im ersten Buch sollen hernach besonders untersucht werden.

Gemeinsame Auslassungen: 119, $2 \pi \alpha \dot{\alpha} \alpha \iota>\mathrm{r}-136,18 \tau \dot{o} \nu \beta \alpha-$

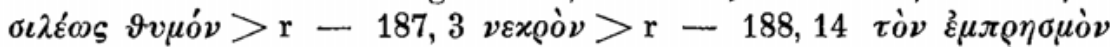

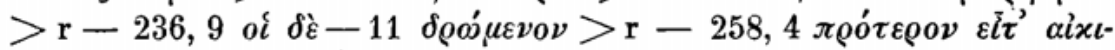

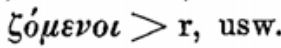

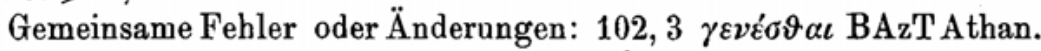

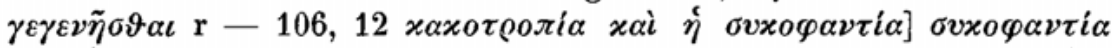

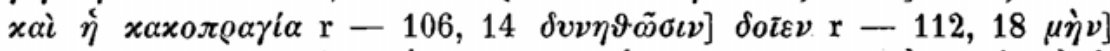

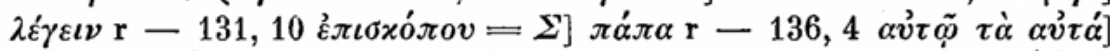

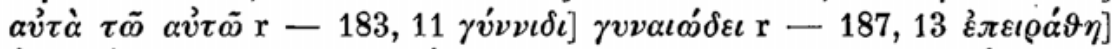

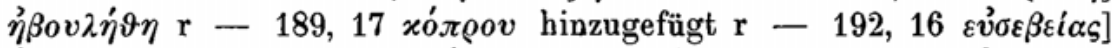

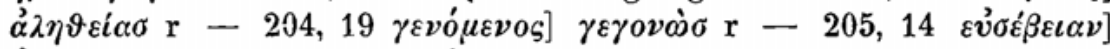

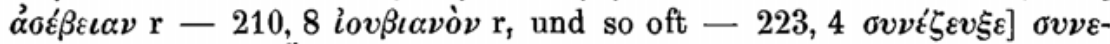
$\zeta \varepsilon v \xi \alpha \mu \varepsilon v$ r, eine Änderung, welche die früheren Herausgeber irregeführt hat. - Eine andere lehrreiche Änderung 225,5 ov $\tau o \tau \sigma \sigma \tau \tilde{\omega} \nu$ i $\mu \varepsilon-$

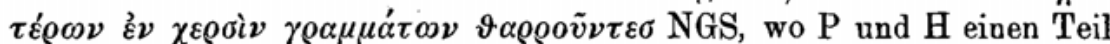
dieser unverständlichen Lesart streichen bzw. auslassen. - 248, 2 nach

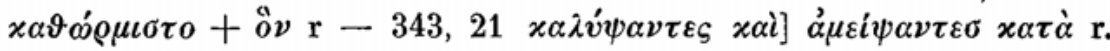

3. Die Einzelgruppen GSP(s) und $H N(n)$ in $r$

Innerhalb der Gruppe $\mathbf{r}$ stellen die Handschriften HN einerseits und GSP anderseits zwei verschiedene Fassungen dar, die ich mit den Siglen $\mathrm{n}$ und $\mathrm{s}$ bezeichnet habe. Von diesen beiden Fassungen ist $\mathrm{s}$, durch GS (stellenweise $+\mathrm{P}$ ) vertreten, die bei weitem fehlerhaftere.

a) Die Gruppe s. Die Auslassungen sind besonders zahlreich:

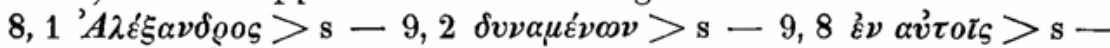

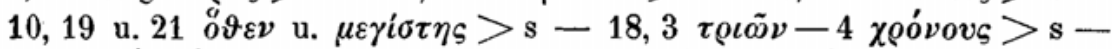

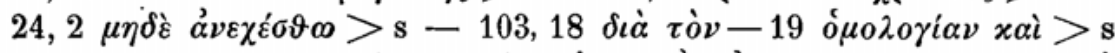

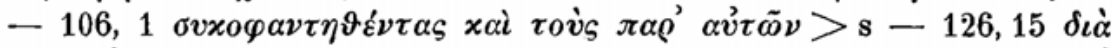
- $\sigma x o ́ \lambda o \pi \alpha \varsigma>\mathrm{s}-130,12 \mu \grave{\varepsilon} \nu-13 \mathrm{Kv} \varrho o v>\mathrm{s}-180,7 / 8 x \alpha \vartheta^{\prime}$

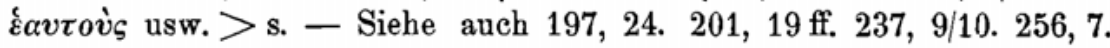
280, 18. $291,16 / 17.303,22 / 23$ usw.

Fehler oder Änderungen: 5, $4 \sigma \tau \rho \varepsilon \beta \lambda \tilde{\omega} \nu] \pi \rho \grave{\sigma} \sigma \beta \sigma \lambda \tilde{\omega} \nu \mathrm{G} \pi \rho \circ \sigma \beta \alpha \lambda$ -

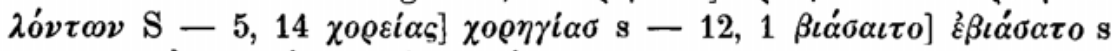

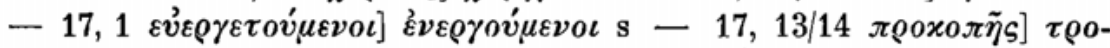

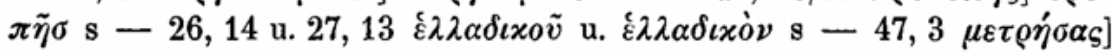




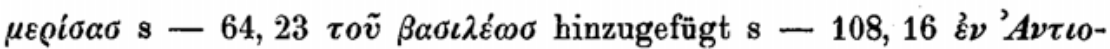

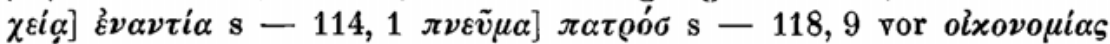
$+\vartheta \varepsilon i \alpha \sigma$ s. - Vgl. auch 174, 7. 179, 11. 182, 14/15. 271, 15. 291, 21. $312,7.329,3$ u. 4.

G. Von den Gliedern der Gruppe $\mathrm{s}$ ist $\mathrm{G}$ das weitaus mangelhafteste. Seine Orthographie wimmelt von Fehlern.

S. Die Handschrift $\mathbf{S}$ ist viel sorgfältiger. Gelegentlich tritt sie aus ihrer Gruppe heraus und führt Correcturen ein, von denen einige das Rechte treffen. $44,11 \delta o x \varepsilon \tau \nu>\mathrm{nG}$, findet sich in $\mathrm{S}-71,12 \mu \eta \delta \dot{\varepsilon}] \mu \grave{\eta} \delta \varepsilon \tilde{\imath} \nu \mathrm{S}$, hier in Übereinstimmung mit Gelasius, der gleichfalls bessert - 103, 2 der Fehler $\lambda \dot{\varepsilon} \gamma \omega$ nG wird von S verbessert in $\lambda \dot{\gamma} \gamma o \mu \varepsilon v$. - Vgl. auch 5, 4. 135, 22. 190, 11. $253,15 / 16$. 265, 18. 296, 7. 328, 1. 336, 1. 338, 1 .

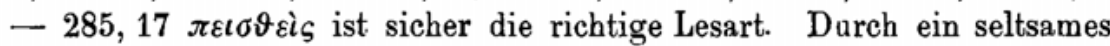
Zusammentreffen begegnet sich $\mathrm{S}$ in der Lesart $\dot{\eta} \sigma \vartheta \varepsilon \dot{\jmath} \sigma$ mit Symeon Vita Ambrosii und mit Cassiodor laetatus. An dieser Stelle ist das Zeichen für einen Absatz handschriftlich bezeugt. Vielleicht ist für Cassiodor der Ursprung des Zusammentreffens ein $\boldsymbol{I}$ gewesen, das der Rubricator in der von Cassiodor benutzten griechischen Handschrift vergessen batte: $\varepsilon \epsilon \sigma \vartheta \varepsilon i c$ erklärte sich der Lateiner als $\eta \sigma \vartheta \varepsilon i$ s. Aber für die Vita Ambrosii läßt ein zweites Zusammentreffen daran denken, daß Symeon eine $\mathrm{S}$ nahestehende Handschrift benutzte: 303, $21 \vartheta \eta \gamma^{\prime}$ $\mu \varepsilon v o \varsigma=\Sigma] \vartheta \varepsilon \lambda \gamma \gamma^{\prime} \mu \varepsilon v o \sigma$ S Symeon.

P. Diese nur als Bruchstück erhaltene Handschrift vom Ende des IX. Jahrhunderts vermeidet natürlich manche Fehler von $G$ und $S$, s. $190,11.199,24.223,21$ usw. Sie stellt jedoch nicht den Arche-

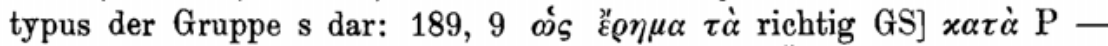

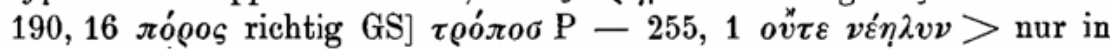
P., usw.

Die gemeinsame Vorlage s zeigte bereits die große Lücke 244/245 und war mit Anmerkungen und Varianten am Rande versehen, s. S. 197, 14. $259,19.268,8$.

b) Die Gruppe n. Den Archetypus n charakterisieren zahlreiche Auslassungen, die $\mathrm{HN}$ allein gemeinsam sind:

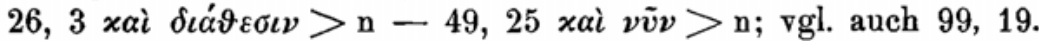
102, 13. 131, 22/23. 132, 4. 151, 2 ff. 193, 4/5. 253, 4. 262, 17. 281, 14/15. $320,23 / 24$.

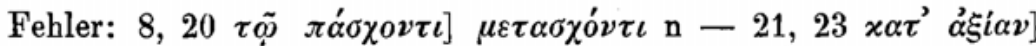

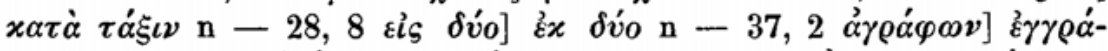

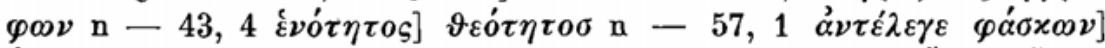

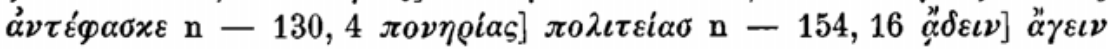




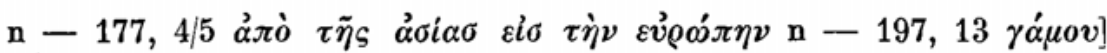
vónov $\mathrm{n}$.

Aber der Sondertypus n ergibt sich auch ans Correcturen oder $\mathrm{Zu}$ sätzen und Einfügungen willkürlicher Worte. Manche dieser Änderungen sind biblischen Ursprungs, z. B.13,13. 64, 23. 225, 8. Beispiele andrer Art

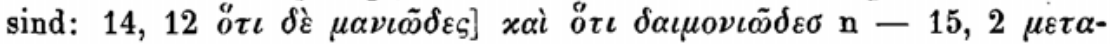
$\gamma \varepsilon \nu \varepsilon ́ \sigma \tau \varepsilon \rho o \nu=$ Cass.] $\mu \grave{\eta} \pi \varrho \sigma \gamma \varepsilon \nu \varepsilon ́ \sigma \tau \varepsilon \rho o \nu \mathrm{n}-16,21 \overline{\iota \sigma} \overline{\chi \sigma}$ hinzugefügt

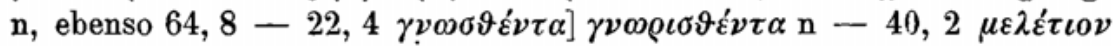
$\varphi \alpha \mu \grave{v}$ hinzugefügt n. - Andre willkürliche Zusätze 57, 7. 61, 16. 81, 11.

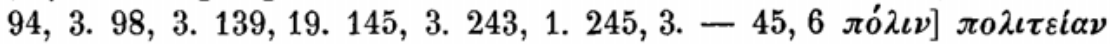

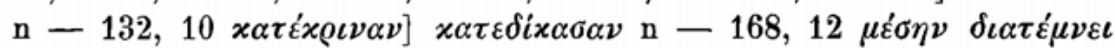

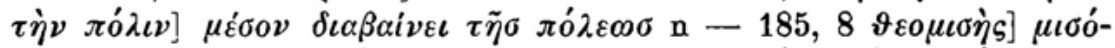

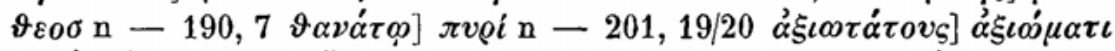

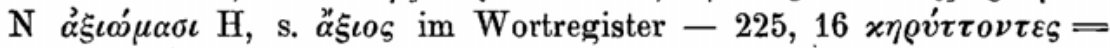
Cass.] $\delta o \xi \alpha \dot{\alpha} \zeta o v \tau \varepsilon \sigma \mathrm{n}$; vgl. 226, 5. 245, 19. 272, 10. 287, 18. 301, 18. 321, 16. 325, 18.

Das Urteil über die Recension n muß der Tatsache Rechnung tragen, daß die Quelle offenbar in ziemlich schlechtem Zustand gewesen ist. Daher kommt es wohl häufig, daß $\mathrm{N}$ und besonders $\mathrm{H}$ unter Aussparung leeren Raums Worte auslassen. Die Divergenz mancher ihrer Lesarten dürfte in den verschiedenen Mitteln ihre Erklärung finden, die sie zur Ausfüllung einer Lücke anwenden, z. B. 180, 16 xoıvovías] $\sigma v \mu \mu$ @i $\alpha \sigma \mathrm{H} \delta v \sigma \sigma \varepsilon \beta \varepsilon i \alpha \sigma \mathrm{N}$, oder in dem Umstande, daß nur in einer der beiden Handschriften der Versuch gemacht ist, die Lücke zu ergänzen: $259,14 / 15$ ov̉x $\eta^{\eta} \nu>\mathrm{N}, \mu \grave{\eta} \mathrm{H}$.

Die Quelle $\mathrm{r}$ selbst war sicher mit Varianten am Rande versehen, unter denen die Wahl verschieden ausfallen konnte. Dafür ein ein-

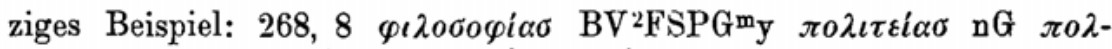

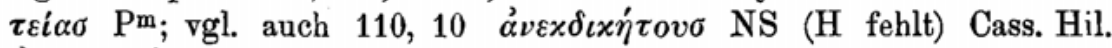

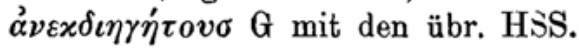

\section{Die Beziehungen $\mathrm{zwischen} \mathrm{n}$ und $\mathrm{s}$ im ersten Buch}

Im allgemeinen gehen im ersten Buch die Überlieferungen von $\mathbf{n}$ und $\mathbf{s}$ so weit auseinander, daß ich darauf verzichtet habe, sie dort zu einer einzigen Gruppe zusammenzufassen.

Sicher findet man selbst in diesem Teil fehlerhafte Lesarten, die an gemeinsamen Ursprung denken lassen: $29,4 \alpha v \tau \tilde{\varphi}=$ Cass. Cand. $>\mathrm{ns}$

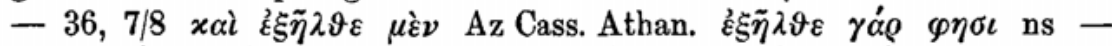

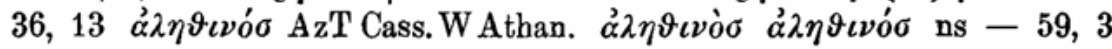
u. 8 stellen die Lesarten $\sigma \omega \tau \tau \tilde{\eta} \varrho \circ \varsigma$ und $\dot{\varepsilon} x \delta \iota \omega \chi \vartheta \vartheta \dot{v} v \tau(\varsigma$, die ns und manchen Handschriften von Eusebius gemeinsam sind, vielleicht alte Varianten 


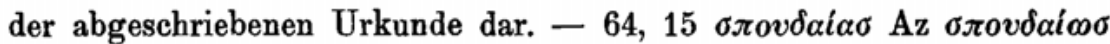

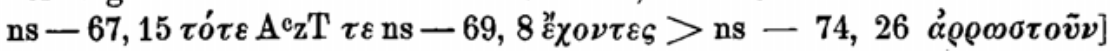

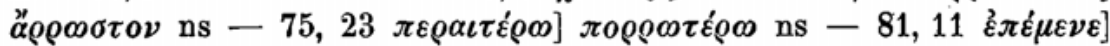

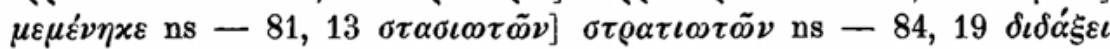
$>$ ns. Die Anzeichen von Verwandtschaft nehmen zu, je weiter man in das Werk hineinkommt.

Anderseits begegnet es gleichfalls in diesem ersten Buch, daß s, sich neben $\mathrm{n}$ stellend, die richtigen Lesarten von A gegen $\mathrm{z}$ teilt, eine wichtige Gruppe, die wir damit wieder zutage treten sehen: $21,17 \pi \varepsilon \pi \omega \rho \infty-$

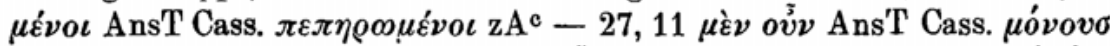

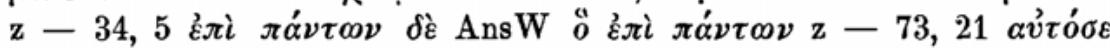

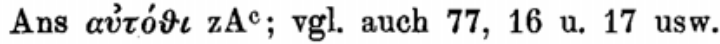

Ebenso endlich wie in der Folge $r$ allein gute Lesarten kann erhalten haben (z. B. 110,5 u. 10;139, 4; 145, 10), scheinen in diesem Teile $\mathrm{n}$ und $\mathrm{s}$ einigemal die bessere Überlieferung gegen $\mathrm{A}$ darzustellen:

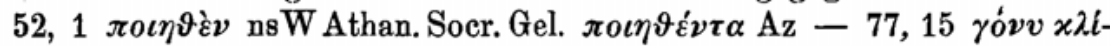

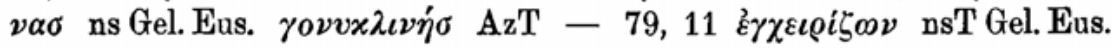
$\dot{\varepsilon} \gamma \chi \varepsilon \iota \rho \zeta \zeta \infty \mathrm{AzW}$.

Trotzdem trennen sich im ersten Buch $\mathbf{n}$ und $\mathbf{s}$ in zablreichen und sehr bezeichnenden Fällen, und dieses Auseinandergehen vollzieht sich immer in der Richtung, daß dann s eine fehlerhafte Lesart der Gruppe z darbietet und innerhalb jener Gruppe selbst besonders $\mathbf{v}$ auf seiner Seite hat.

Gute Lesarten von An stehen Fehlern von sz gegenüber: 4, $15 \tau \tilde{\eta}$ $\ldots \delta v v \alpha ́ \mu \varepsilon \iota$ An $\tau \dot{\eta} v \ldots \delta \dot{v} \alpha \mu \iota v \mathrm{sz}-5,7 \tau \alpha \dot{v} \tau \eta \sigma \mathrm{An}>\mathrm{sz}-5,14$ $\varepsilon \dot{\mu} \mu \pi \lambda \varepsilon \alpha$ An Eus. $\alpha \dot{\alpha} \alpha \dot{\pi} \pi \varepsilon \varepsilon \alpha \mathrm{sz}-6,15 \delta \dot{\mathrm{AnT}} \tau \varepsilon$ sz. - Vgl. 9, 8. 10, 1.

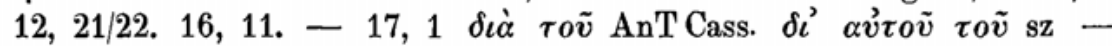
24, $7 \dot{\eta} \pi \alpha \tau \eta \mu \varepsilon ́ v \alpha] \pi \varepsilon \pi \lambda \alpha \nu \eta \mu \varepsilon ́ v \alpha$ sz. $-24,17$ bildet der Name des

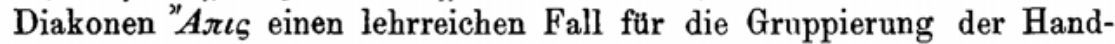
schriften. Der Genitiv "A $A \iota$ in An ist die Form des Archetypus. T (= Cass.), durch die Form wohl in Verlegenheit gesetzt, läßt den

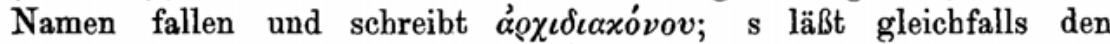

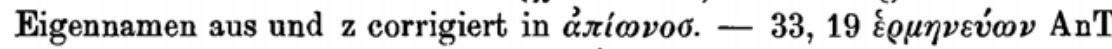

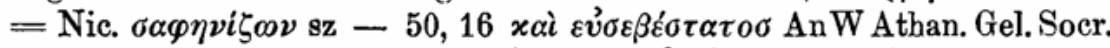

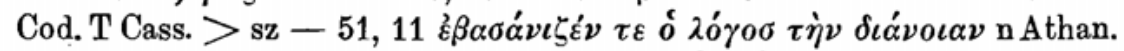

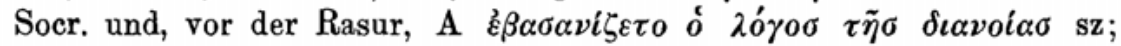
vgl. auch 52, 13. 53, 8 u. 21/22. 54, 10 usw.

Innerhalb der Gruppe $\mathrm{z}$ schöpft $\mathrm{s}$ seine fehlerhaften Lesarten aus einer Handschrift des Zweiges $\mathrm{v}: 4,7 \stackrel{\alpha}{\nu} \vartheta \vartheta \tilde{v} \sigma \alpha \nu$ AnDL $\dot{\alpha} \nu \grave{\alpha} \pi \tilde{\alpha} \sigma \alpha \nu$ sv $-4,14 \delta \dot{\eta}>\mathrm{sv}-8,7 \pi \lambda \alpha \sigma \tau \tilde{\omega} \sigma \tau \alpha \tilde{v} \tau \alpha$ sv. $-10,4 / 5 \mathrm{sF}$ stimmen in derselben fehlerhaften Änderung überein, die V aufs neue ändert; 
vgl. auch 11, 16. 14, 13. 52,10. - 43, 19 xoเvòv $>\mathrm{sv}-55,9 / 10 \chi \alpha i$ ○⿻v $\delta \grave{\eta} \mathrm{sv}$.

Diese Verwicklung in den Beziehungen zwischen $\mathbf{n}$ und $\mathbf{s}$ besonders im Anfang läßt verschiedene Möglichkeiten der Erklärung zu; vgl. z. B. wie de Boor ein ähnliches Problem in Theophanis Chronographia II $527 \mathrm{ff}$ untersucht hat. Soll man annehmen, daß für diesen Teil $\mathbf{s}$ aus einer Quelle geflossen ist, welche von der Quelle von $\mathbf{n}$ völlig verschieden ist? Das scheint mir mit Räcksicht auf die dargelegten Übereinstimmungen von n mit s (S. XXVf) kaum möglicb. Ohne alle hier denkbaren Hypothesen zu erörtern, will ich nur auf die folgende aufmerksam machen. Während des ganzen Zeitabschnittes der Textgeschichte, den wir beobachten können, hat die fehlerhafte Recension, die für die Überlieferung $\mathrm{z}$ charakteristisch ist, das Bestreben gehabt, die andern Handschriften zu beeinflussen und die ältere Überlieferung in ihnen zu verdrängen. Der Fall von $A^{c}$ ist dafür ein schlagendes Beispiel, und wir werden noch andre Beweise kennen lernen. Notwendig muß man das Vorhandensein alter Handschriften annehmen, in denen sich Lesarten zweier verschiedener Klassen fanden. Bei dieser Voraussetzung hat s von Anfang an in größerer Zahl Lesarten der Klasse $\mathrm{v}$ aufgenommen, während n sie mehr vermied. Ein Fall, bei dem man gewissermaßen mechanisch in $\mathrm{s}$ den Fehler von $\mathrm{z}$ an die Stelle der richtigen Lesart treten sieht, ist: 22, $1 \pi \alpha \varrho \dot{\alpha} \tau \tilde{\omega} \nu \stackrel{\alpha}{\alpha} \operatorname{\alpha o\alpha } \tau \tilde{\omega} \nu$ nT Cass. und, vor der Rasur, A $\delta \iota \dot{\alpha} \tau \tilde{\omega} \nu$

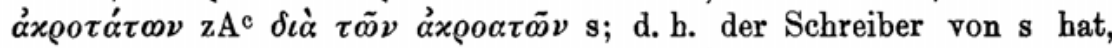
nachdem er in seinem Text $\pi \alpha \rho \alpha \dot{~ d u r c h ~} \delta i \dot{\alpha}$ aus z ersetzt hatte, vergessen nun auch noch $\alpha$ xoo $\alpha \tau \tilde{\omega} \nu$ zu bessern.

Wir werden aus Fällen, wo allein $\mathrm{A}$ oder allein $\mathrm{AB}$ gegen alle andern Handschriften die richtige Lesart haben, alsbald (unten S. XXXIIf) sehen, daß sogar n selbst schon von Anfang an sich nicht ganz den Lesarten

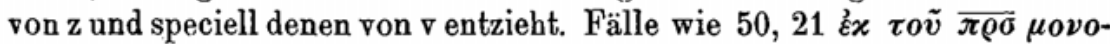

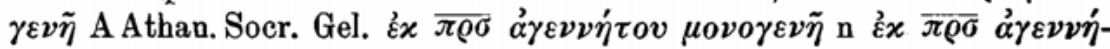
cov sz zeigen gut, wie $\mathrm{n}$ und $\mathrm{s}$ sich in verschiedener Weise von den Varianten ihres Archetypus beeinflussen lassen. Je weiter das Werk fortschreitet, um so mehr stimmt $\mathbf{n}$ in diesen falschen Lesarten mit $\mathbf{s}$ überein (s. den umgekehrten Fall in v, S. XXXV u. XXXIXf): 65, 23

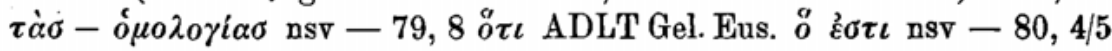

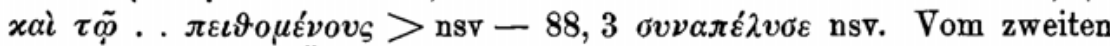
Buch $\mathrm{ab}$ ist die Übereinstimmung von $\mathrm{n}$ und $\mathrm{s}$ ( $\mathrm{r}$ ) im allgemeinen constant, sei es in den Abweichungen zugunsten der Gruppe v, z. B. $110,11.144,12$ u. $23 \mathrm{f}$. $154,3.155,1$, sei es in den richtigen Lesarten, z. B. $119,22.126,11.132,4 / 5$ u. $16.134,16,18$ u. $22.135,20$. 138, 6 usw. 


\section{Die Beziehungen von $\mathrm{n}$ zu $\mathrm{A}$ und $\mathrm{T}$}

Trotzdem ist es von Wichtigkeit zu bemerken, daß der besondere Wert von $n$ gegenüber $\mathbf{s}$ sich noch vereinzelt in dem ganzen weitern Verlauf des Werkes bekundet. n stellt nach wie vor zuweilen eine Quelle dar, die besondere Beziehungen zu A hat. Diese Verwandtschaft von n mit dem Zweig A zeigt sich zunächst in charakteristischen gemeinsamen alten Fehlern.

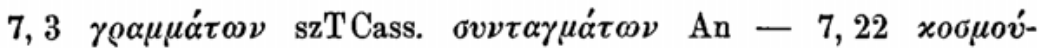

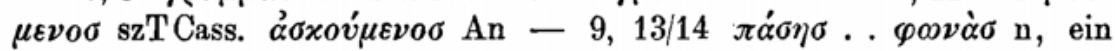
alter Fehler, der, scheint es, auch in A vor der Correctur vorhanden

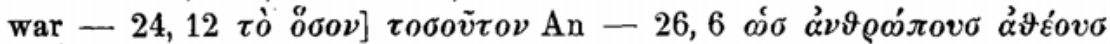

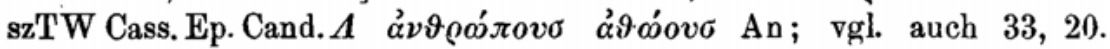

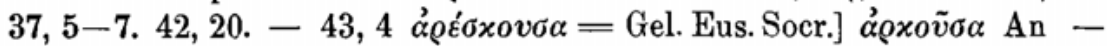

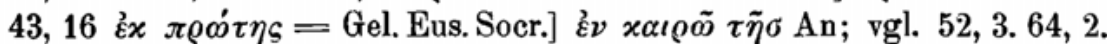

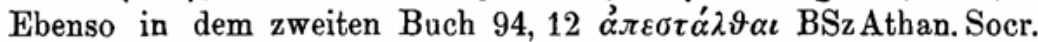

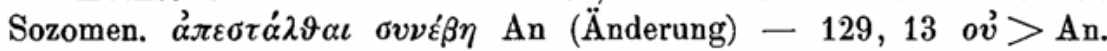
Zum Schluß endlich noch der sehr beweiskräftige gemeinsame Fehler 144,5 in $\varepsilon \dot{v} \sigma \varepsilon \beta \varepsilon i \alpha$ oov $\mathrm{szA}^{\mathrm{c}} \tau \tilde{\eta} \sigma \quad \varepsilon \dot{v} \sigma \varepsilon \beta \varepsilon i \alpha \sigma$ ovंx An.

Anderseits hat $\mathrm{n}$ sich dank seiner vortrefflichen Quelle einigemal von Änderungen frei gebalten, die selbst A ergriffen haben, und in diesem Fall wird seine Lesart mehrfach von T bestätigt, z. B. 12, 19 ov $\gamma \dot{\alpha} \rho$ $\pi \omega \sigma \quad \delta$ nT ov $\gamma \dot{\alpha} \varrho \pi \tilde{\alpha} \sigma \mathrm{Asz}$; vgl. 22, 1 u. 22. 26, 15. - 62, $4 \stackrel{\circ}{\sigma} \sigma \omega$ nTW Socr. Eus. - 71, $13 \ddot{\eta}$ nT Gel. Nic. aut Cass. xaì Asz.

Diese Quelle von $\mathrm{n}$ hatte - und wir werden sehen, daß es mit A ebenso steht - besondere Beziehungen zu der Quelle von T, wie gewisse gemeinsame falsche Lesarten beweisen; vgl. besonders 34, $14 \pi \varrho 0 \varepsilon \delta \varrho \iota \tilde{\omega} \nu]$ $\pi \rho o z \delta \rho \omega \nu$ nT; das Alter des Fehlers in T wird bezengt durch praesulibus Cass. - 61, $21 \lambda \alpha \vartheta \varepsilon \tau \nu$ szW A ${ }^{\circ}$ Socr. Eus. $\varepsilon^{\prime \prime} \lambda \alpha \vartheta \varepsilon v$ AnT, und 23

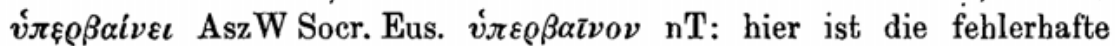
Übereinstimmung mit $\mathrm{T}$ von $\mathrm{A}$ beseitigt, dagegen von $\mathrm{n}$ erhalten worden; s. auch 67,2 हैं $\varepsilon \varepsilon \mu \psi \varepsilon$ nT.

Selbst über das erste Buch hinaus fährt $\mathrm{n}$ fort, hier und da vortreffliche Lesarten zu bewahren, während s Varianten aufnimmt, die wohl am Rande der Quelle standen. In dieser Hinsicht dürfte der kritische Apparat der Seite 148 als besonders lehrreich zu gelten haben:

148, 2 v่ต' die übr. HSS und Sozomenus. Wegen Sozomenus, von dem aus eine Beeinflussung der Theodoret-Überlieferung sicherlich nicht stattgefunden hat, betrachte ich die Variante als bereits in der Sammlung selbst vorhanden, aus der die Urkunde entnommen ist. Ebenso sind die böchst 
merkwürdigen Varianten zu beurteilen, die sich auf der gleichen Seite $3 / 4,7 / 8,9$ usw. finden. Der Archetypus dieser Urkunde war mit Varianten versehen, die zum Teil in die ersten Abschriften übergegangen sind und hernach verschiedene Schicksale gehabt haben. Unter andern ähnlichen Fällen vgl. z. B. 104, 15. 105, 17/18. 220, 12. 225, 11.

\section{Der Wert von $\mathrm{H}$}

In der Gruppe $\mathbf{n}$ bietet $\mathrm{H}$ ein Problem, das vor der Aufstellung des Textes der Lösung bedarf. $\mathrm{H}$ steht nämlich mit einer großen Zahl von Lesarten allein, welche die frühern Herausgeber bestochen haben und so seit Estienne und Sirmond vielfach in den Textus receptus übergegangen sind. Es kommt nun darauf an festzustellen, ob gewisse Lesarten wirklich auf die gute Überlieferung zurückgehen, durch die die ganze Gruppe n bisweilen sich auszeichnet.

Es sei sogleich verraten, daß diese $\mathrm{H}$ eigentümlichen Lesarten fast immer nur geschickte Verbesserungen oder willkürliche Änderungen sind. Im allgemeinen genügt ein Blick in den kritischen Apparat, um den Nachweis zu führen.

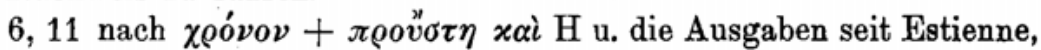
gegen TCass. u. die übr. HSS.

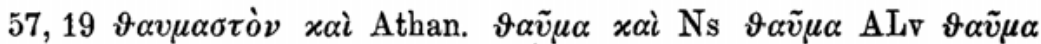

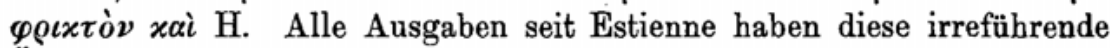
Änderung angenommen. In Wirklichkeit haben allein Ns mit xaì die Spur der Lesart des Athanasius erhalten. Während ALv xai wegen des Fehlers in $\vartheta \alpha \tilde{v} \mu \alpha$ fallen liessen, hat der Schreiber von $\mathrm{H}$ sich die Sache zurecht-

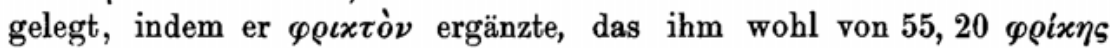

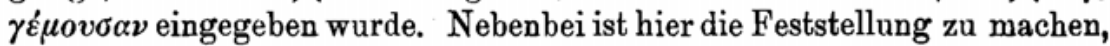
daß dieser geschickte Emendator nicht daran denkt, Atbanasius zu Rate zu ziehen, sondern mit eigenen Conjecturen vorgeht.

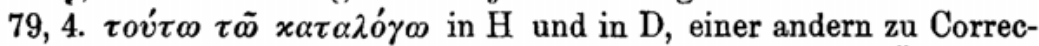
turen neigenden Handschrift, kann ich nur als eine erleichternde Änderung

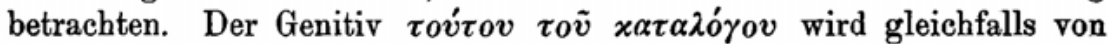
allen Handschriften des Eusebius dargeboten, und Heikel würde ihn wohl beibehalten haben, wenn er über die Überlieferung des Theodoret besser unterrichtet gewesen wäre. Der gleiche Fall scheint mir zwei Zeilen weiter 79,6 vorzuliegen, wo ich beibehalten habe ${ }^{\circ} \tau \alpha \nu=$ quando Cass. o $\tau \varepsilon \varepsilon$ Eus. Cod. $V$ gegen die Correctur ${ }^{\circ} \tau \iota$ HD und die minderwertigen Handschriften des Eusebius, denen Heikel gefolgt ist.

Die Lesart von $\mathrm{H} \alpha^{\alpha} \xi \iota \omega^{\prime} \mu \alpha \sigma \iota 136,14$ u. 246, 17 ist eine Correctur, welche die Herausgeber seit Estienne zu Unrecht aufgenommen haben; vgl. Wortregister s. v. $\alpha^{\prime} \xi \iota 0$. 
140, 12 nach ${ }_{\alpha}^{\prime} \tau o \pi o \nu+\dot{\varepsilon} \nu o \mu i \sigma \alpha \mu \varepsilon \nu \mathrm{H}$ und die Ausgaben seit Estienne. Athanasius, Socrates und Sozomenus haben keine Spur von dieser Einschiebung, die übrigens geschickt ist und in einen umständlichen Satz Klarheit bringt. Aber $\mathrm{H}$ verrät sich doch als Corrector durch eine weitere Änderung, die er auf der vorhergehenden Zeile hat vornehmen müssen (s. App. 140,11). Incongruum esse judicamus in Cass. ist keine Bestätigung von H. Jeder Übersetzer geht darauf aus, einen verständlichen Text zu geben, und sieht sich, so gewissenhaft er auch sein mag, zuweilen gezwungen, auszulegen und zu umschreiben. Wie der Zusatz sich an dieser Stelle gleichsam von selbst darbot und wie leicht das Zusammentreffen war, beweist Hilarius, der sonst eine ziemlich weit abliegende Fassung darstellt, mit seinem nefas putamus.

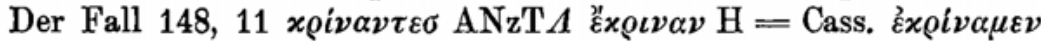
Sozom. muß genau ebenso eingeschätzt werden. Vgl. auch 255, $1 \sigma \varepsilon$ $\beta o v \tau \varepsilon \varsigma] \sigma \varepsilon ́ \beta \beta \mu \varepsilon v$ H colimus Cass.

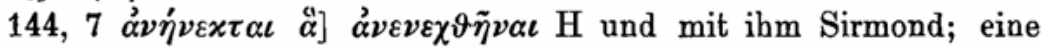
geschickte Conjectur, die eine in traurigem Zustand befindliche Urkunde verbessern würde.

Etwas weiter unten hat in derselben Urkunde 144, 12 der Archetypus $r$ in Übereinstimmung mit andern Handschriften das unentbehr-

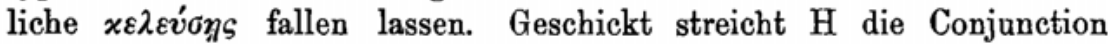
Iv $\alpha(144,11)$, von der $x \varepsilon \lambda \varepsilon v \dot{\sigma} \eta \eta_{\varsigma}$ abhing, und stellt so einen verständlichen Satz her. - Vgl. für andre willkürliche Correcturen 163, 22. 256, 8. 257, 17/18. 259, 23. 265, 2. Eine große Zahl von ihnen, die ich nicht verzeichnet habe, ist in die Ausgabe von Estienne und besonders in die von Sirmond übergegangen.

$256,16 \delta v \sigma \sigma \varepsilon \beta \varepsilon i \alpha]$ ai $\varepsilon^{2} \sigma \varepsilon \iota \mathrm{H}>\mathrm{N}$ : das Wort war in n ausgefallen;

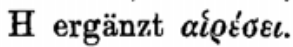

257, 7 ändern $\mathrm{H}$ und mit ihm die Herausgeber seit Estienne die ungebräuchliche Form $\alpha^{\prime} \lambda \lambda \varepsilon \pi \alpha^{\prime} \lambda \lambda \eta$ in $\alpha^{\prime} \lambda \lambda \varepsilon \pi \alpha \lambda \lambda \eta^{\prime} \lambda \omega$.

Selbst gewisse Auslassungen sind lehrreich, um die Arbeitsweise von H zu charakterisieren. 134, 23 bieten die Handschriften seiner Gruppe Ns die unmögliche Lesart v́ $\varphi \eta \gamma \eta \sigma \alpha ́ \alpha \varepsilon v o \iota . ~ H$ läßt den Platz für das ihm nicht verständliche Wort leer; vgl. 225, 5 .

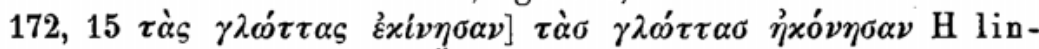
guas exacuerunt Cass. Die Übereinstimmung mit Cass. läßt daran denken, daß sich $\mathrm{H}$ hier eine sehr alte Variante aneignet, die durch ähnliche Anwendungen des Verbs áxováo inspiriert ist, z. B. Psal. 140, 4. Socrat. III 12, 7. Theodoret selber muß geschrieben haben $\dot{\varepsilon} x i-$

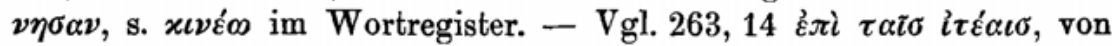
$\mathrm{H}$ in den Text eingeführt nach Psal. 136, 2. 


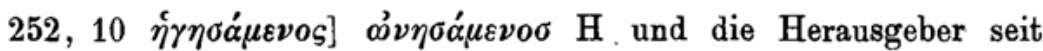
Estienne. Eine geschickte Correctur, welche die lectio difficilior verschwinden läßt; wie auf S. 172, 15 ist diese H-Lesart vielleicht alt, vgl. comparans Cass.

Beispiele wie die beiden letzten legen den Gedanken nahe, daß unter den im allgemeinen verdächtigen Lesarten von $\mathrm{H}$ möglicherweise dennoch einige von altem Ursprung begegnen. In der Tat sind manche von ihnen wertvoll und gehen auf die gute Überlieferung zurück, die häufig im ersten Buch die ganze Gruppe $\mathbf{n}$ mit A darstellte. In dieser Hinsicht ist eine fehlerhafte Übereinstimmung wie 148, 6 o $\pi \lambda \omega \nu=$ Sozom. Cass. A] ڤڤov AH sehr lehrreich, denn sie zeigt uns einen sichern Fall, wo allein $\mathrm{H}$ mit der Quelle von A zusammengeht.

Aus dieser Quelle nun schöpft der Codex $\mathrm{H}$ einige gute Lesarten, die er uns allein erhalten hat:

$103,7 \mathfrak{z} x \tau o \tilde{v}$, durch die ganze Parallelüberlieferung, Athanasius $A$ Hilarius, bestätigt, wird in der Überlieferung des Theodoret nur von $\mathrm{H}$ geboten. Die Annahme, daß $\mathrm{H}$ nach Athanasius abgeändert hätte, halte ich für gänzlich ausgeschlossen. Wir haben oben (S. XXIX) gesehen, wie er willkürlich einen Fehler gebessert hat, den er bei einem Blick auf den Text des Athanasius vermieden haben würde. Unter den zahlreichen Lesarten dieses geschickten Emendators spricht nicht das geringste Anzeichen für eine Revision nach fremden Quellen. Ich lege weiter unten dar, daß eine solche Arbeitsmethode den Verfassern unsrer Handschriften überhaupt nicht in den Sinn gekommen ist. Für gleichfalls unwahrscheinlich halte ich es, daß H so glücklich gewesen wäre, durch Conjectur die richtige Lesart wiederzufinden. $\mathrm{H}$ hat also an dieser Stelle die gute Überlieferung treuer als $\mathrm{A}$ und $\mathrm{N}$ bewahrt.

Diese Auffassung wird durch eine andere richtige Lesart von $\mathrm{H}$ bestätigt, die uns ein kurzes Stück dahinter begegnet:

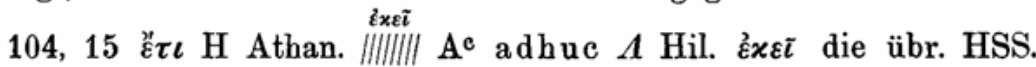
Hier liegt die deutliche Spur einer alten Doppellesart vor, deren bessere Fassung nur $\mathrm{A}$ und $\mathrm{H}$ erhalten haben.

Demzufolge darf man darauf gefaßt sein, daß $\mathrm{H}$ im ganzen Verlauf des Werkes zuweilen die Fehler seiner Gruppe $r$ vermeidet, indem er die Spur von richtigen Lesarten seiner Quelle bewahrt, ohne daß von selbständiger Änderung die Rede sein kann: 224, 12 x $\alpha \rho \circ \varphi \varrho v \gamma i \alpha \sigma$ $\mathrm{BV}^{2} \mathrm{HW}$ Cass. $>$ NsyF; vgl. 227,4 u. 9. - 240, $9 \pi \rho \varepsilon \sigma \beta \dot{v} \tau o v B{ }^{2} \mathrm{HNic}$.

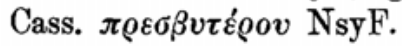




\section{Der Wert von A}

Im Vorhergehenden ist der Wert von $\mathbf{A}$ bereits hinsichtlich seiner Beziehungen zu $\mathrm{n}$, zu $\mathrm{H}$ und $\mathrm{r}$ und auch zu $\mathrm{A}^{\mathrm{c}}$ untersucht worden.

Wie ich (S. XXVIII) auf besondere Beziehungen zwischen $\mathrm{n}$ und T aufmerksam gemacht habe, so besitzt der mit $\mathrm{n}$ verwandteCodex $\mathrm{A}$, wie man erwarten durfte, ähnliche Beziehungen zu jener Abschrift der Tripartita.

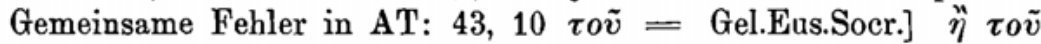
$\mathrm{AT}-62,16 \delta \iota \alpha \tau \alpha^{\prime} \xi \alpha \iota \tau \varepsilon=$ Gel.Socr.Eus.] $\delta \iota \alpha \tau \alpha^{\prime} \xi \alpha \sigma \vartheta \alpha \iota \mathrm{T}$ und, vor der Rasur, A - 78, $15 \mu \tilde{\eta} \nu \iota \mathrm{G}=$ Eus. Sozom.] $\mu \dot{\eta} \nu \eta \sigma \iota \sigma \mathrm{A} \mu \dot{\eta} \nu \iota \sigma \iota \sigma \mathrm{T}-$

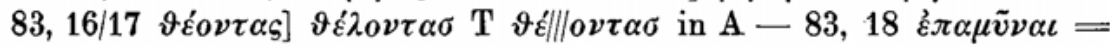
Eus.] $\dot{\varepsilon} \pi \alpha \mu \tilde{v} v \alpha \iota \tau \grave{\varepsilon}$ ATGel. Man wird weiter unten sehen, daß Gelasius eine T nahestehende Handschrift benutzt hat; vgl. auch 61, 21. 82, 7.149, 1.

$\mathrm{Zu}$ diesen alten Fehlern fügt A seine besonderen Fehler oder Änderungen hinzu. Die Auslassungen sind ziemlich häufig: 18, 5/6. 25, 11. 27, 2. 43, 14/15. 46. 4. 48, 1. 51, 20. 60, 9. 64, 12. 67, 14. 110, 2. 149, 13 usw.

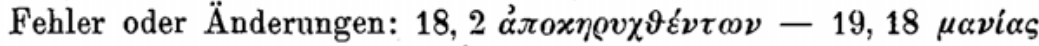
$=$ Cass.] $x \alpha x i \alpha \sigma \mathrm{A}-43,11$ hó $\omega \omega=$ Gel.Eus.Socr.] $\lambda \sigma \gamma \iota \sigma \mu \tilde{\omega} \mathrm{A}-$ $52,16 \stackrel{\alpha}{\alpha} \varphi \omega \mu \circ \iota \tilde{\omega} \sigma \vartheta \alpha \iota=$ Athan. Socr.] ${ }_{0}^{\circ} \mu o \iota v \nu \mathrm{A}-54,13 \pi \dot{\varepsilon} \lambda \alpha \sigma \mathrm{nsz}$

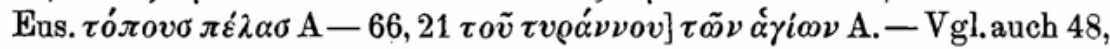
15. 54, 1. 63, 4. 64, 15. 73,10/11. 140,14/15. - Manche allein in A vorkommenden Lesarten, die ich in den Text nicht aufgenommen habe, scheinen sehr beachtenswerte alte Varianten zu sein: $113,17 \mathrm{f} \pi \nu \varepsilon \tilde{v} \mu \alpha$ ovंx $\tilde{z} \chi \propto \nu ;$ vgl. $65,5.114,3$.

Zahlreich sind die guten Lesarten, mit denen A allein steht, unterstützt von der Tripartita oder von der Parallelüberlieferung: 43, 9 ध̇ $\pi \iota \tau \varepsilon \lambda \varepsilon \tau \nu$

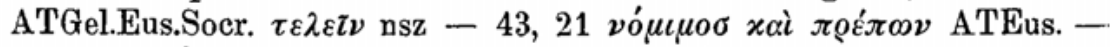

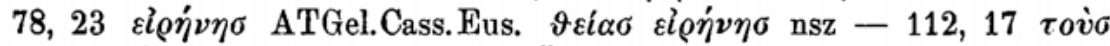

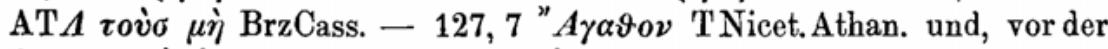

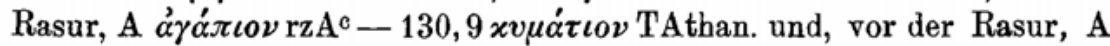

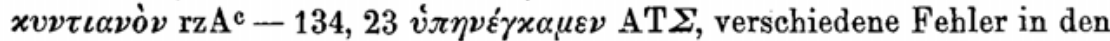
andern Handschriften. Vgl. auch 37, 1. 45, 18. 68, 22. 126, 8. 127; 2. 148, 13/14. 150, 17. 151, 5 usw.

Die Beziehungen zwischen A und B. Der beste Vertreter der ersten Klasse, B, fehlt uns für einen großen Teil der beiden ersten Bücher. Dieser Verlust wird gerade durch die Lesarten der ersten Hand von A einigermaßen gutgemacht. Denn von den ersten Zeilen ab, wo der Codex B erscheint, tritt deutlich zutage, daß er eine weit zurückliegende, dem Archetypus von A ähnliche Quelle darstellt. Zum Beweise dafür einige charakteristische gemeinsame Fehler:

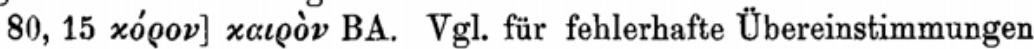


in der Orthographie, die auf die Quelle zurückgehen, 80, 16. 82, 11.

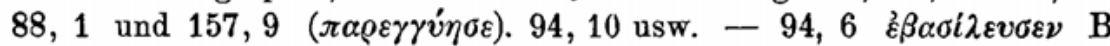

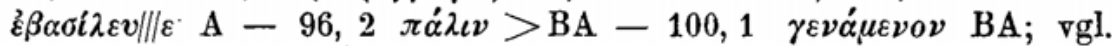

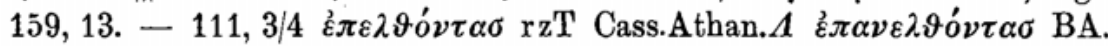
- Vgl. auch 104, 12. 153, 15. 159, 19. 160, 1. Natürlich hören diese Übereinstimmungen auf, sobald A sich einer mit L verwandten Handschrift anschließt.

Ich verzeichne hier nur eine kleine Zahl der hänfigen Fälle,

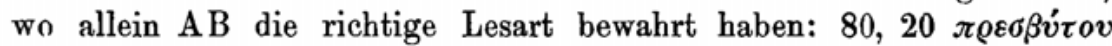

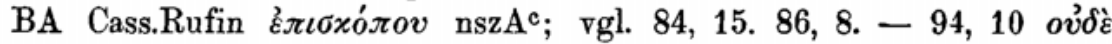

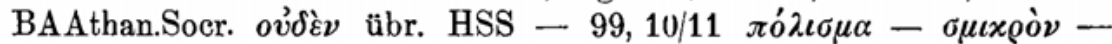

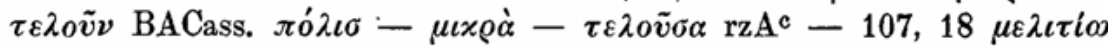
BAAthan. $\Lambda$ Hil. $\mu \varepsilon \lambda \varepsilon \tau i o$ rzT; 117,1 vermeiden BA mit Cass. $\Lambda$ allein

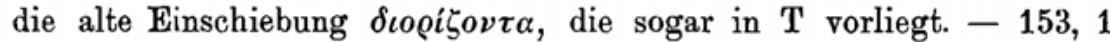

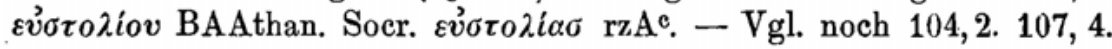
109 , 14. 116, 9. 160, 20 usw.

Auch von solchen Fällen sind einige Beispiele zu geben, wo die Lesart von A allein den Vorzug verdient, selbst wenn TBCass. fehlen oder abweichen:

50, $21 \dot{\varepsilon} x \tau o \tilde{v} \pi \overline{\varrho \sigma} \mu o v o \gamma \varepsilon v \tilde{\eta}$ AAthan. Socr.Gel., s. ob. S. XXVII -

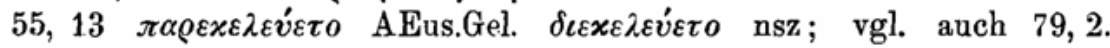

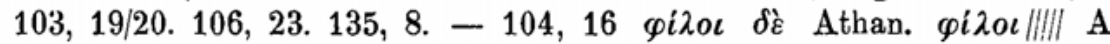

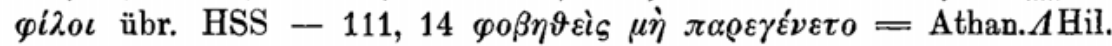
Sozom. ist die gute Lesart, die allein A vor der Rasur gegen den Fehler dei anderen Handschriften, einschließlich B und Cassiodor, bewahrt hatte.

Fa!le wie diese haben mich bestimmt 112, 19 die vortreffliche Lesart

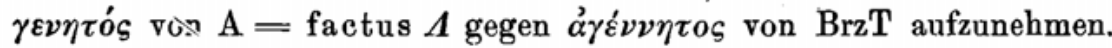
In solchen Fällen handelt es sich augenscheinlich um sehr alte Varianten, die wohl auf den Archetypus der Urkunde zurückgehen; vgl. einen ähnlichen Fall 104, 15 und S. XXVIII f, XXXII, XXXIV und XL.

135, 18 hat mir der Text von Cass. erlaubt, für A die Imperative wiederherzustellen, die $\mathrm{A}^{\mathrm{c}}$ im Anschluß an die andern Handschriften hatte verschwinden lassen.

8. Die Gruppe $D^{2} B^{3} L F V R=z$. Die Handschriften $D B^{2} B^{3} L$

Die Feblerhaftigkeit der Überlieferung in der Gruppe $\mathrm{z}$ ist genügend außer Zweifel gesetzt durch die zablreichen Fälle, wo sie, wie wir gesehen baben, sich den guten Lesarten von BAns (vereint oder getrennt) gegenüberstellt (S. XXI, XXVI, XXXIIf). Es bleiben einige Bemerkungen zu machen über die Handschriften, in welche diese Gruppe sich ihrerseits verzweigt. 
D. Diese junge Handschrift kommt nur für das erste Buch in Frage. Sie wimmelt von Fehlern und willkürlichen Änderungen. Ich habe damit natürlich den kritischen Apparat nicht belasten können. Die Grundlage der Überlieferung von $D$ steht $L$ sehr nahe, aber $D$ hat noch eine andere Handschrift zu Rate ziehen können und vermeidet ziemlich häufig

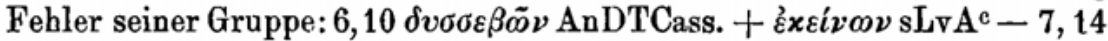
$\tau \tilde{\eta} \sigma$ AnDT Cass. $\tau \tilde{\eta} \sigma \alpha v \dot{v} \tilde{\eta} \sigma$ sLvA; ;gl. auch 5, 24. 8, 7 u. 18. 44, 10. 45, 13. 81, 13. 88, 6. Die Lesart $\alpha \dot{v} \tau \tilde{\eta} 5,7$ darf als eine gute Correctur betrachtet werden.

Anf die Änderungen von D komme ich bei Gelegenheit der Ausgabe Sirmonds kurz zu sprechen. Sie verdienen tatsächlich nur deshalb einiges Interesse, weil Sirmond sie zuweilen in seinen Text eingeführt hat.

$B^{2}$ und $B^{3}$. Zwei verschiedene Hände haben in $B$ verloren gegangene Seiten des zweiten Buches ergänzt. Beide schreiben eine L sehr nahestehende Handschrift ab.

$\mathbf{B}^{2}$ mit nachlässiger Schrift und vielen Abkürzungen ist voller Fehler, die ihm eigentümlich sind, und zeigt außerdem fast alle charakteristischen Fehler der Gruppe z; s. 126, 11. 127, 16. 129, 8. 132, 4/5 u. $16.134,16,18 / 19$ u. $22.135,20$ usw.

Ich habe jedoch den Codex $\mathrm{B}^{2}$ nicht beiseite lassen können, denn er trennt sich, ebenso wie $\mathrm{D}$ im ersten Buch, zuweilen von der Gruppe $\mathrm{z}$ in guten

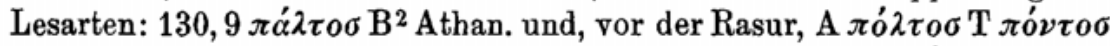

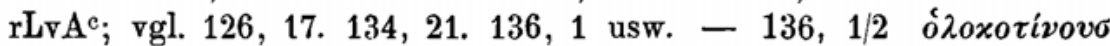

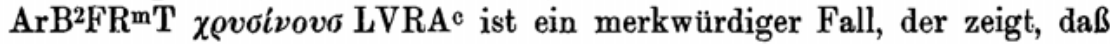
die Quelle z die doppelte Lesart hatte.

$\mathrm{B}^{3}$ schreibt wahrscheinlich dieselbe Handschrift ab wie $\mathrm{B}^{2}$, denn seine Prüfung führt zu den gleichen Schlüssen. $\mathrm{B}^{3}$ hat nicht die besonderen Fehler von $\mathrm{v}$ und ist sehr nahe verwandt mit L: $\left.142,19 \mu \eta^{\prime} \tau \varepsilon\right]$

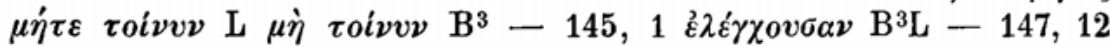
$\left.\pi \alpha^{\prime} \nu v\right] \pi \alpha^{\prime} \lambda \iota v \mathrm{~B}^{3} \mathrm{LA}^{\mathrm{c}}$. Ebenso wie $\mathrm{B}^{2}$ vermeidet $\mathrm{B}^{3}$ gewisse Fehler und Auslassungen von $L v$ : $139,3,13$ u. 21. 140, 1, 5 u. 8. 141, 14/15. 149, 5.

Unter den Lesarten von $\mathrm{B}^{3}$ begegnen ebenso wie bei $\mathrm{B}^{2}$ solche, die von alten Varianten oder Correcturen der Quelle herrühren: 138, 6 x $x o \tau \varepsilon \chi v i \alpha \sigma \mathrm{ArT}$ am Rand $\mathrm{B}^{3} \mu \alpha v i \alpha \sigma \mathrm{z}$. In der Lesart $\sigma \chi \dot{\eta} \mu \alpha \tau o \sigma$ stimmt $\mathrm{B}^{3}$ 148, 8 mit Sozom. Cod. V überein; die andern Handschriften haben

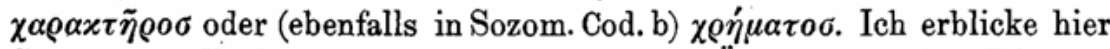
Spuren von Varianten in der ursprünglichen Übersetzung der Urkunde. Bemerkt sei, daß häufige Übereinstimmungen mit Sozomenus bei Varianten der Urkunde S. $147 \mathrm{ff}$ den Gedanken nahelegen, daß Sozomenus bereits in seiner Abschrift Varianten der Gruppe B ${ }^{3} \mathrm{~L}$ vorfand. 
L. Um eine Vorstellung von den speciellen Fehlern von L zu geben, genügen die S. XXI zusammengestellten Beispiele von Fehlern, die $\mathrm{A}^{\mathrm{e}}$ mit $\mathrm{L}$ gemein hat. $\mathrm{L}$ ist übrigens weniger verderbt als $\mathrm{v}$; siehe z. B. 4, 7. 12, 20. 143, 8. 144, 12 usw.

\section{Die Einzelgruppe FVR (v) in z}

$\mathrm{F}$ und $\mathrm{V}$ stellen bis zu dem Augenblick, wo die zweite Hand von V (S. 187) erscheint, dieselbe Überlieferung dar, und zwar bei weitem die verderbteste. $\mathrm{Zu}$ den Fehlern der Gruppe $\mathrm{z}$ fügt $\mathrm{v}$ noch viele ihm eigentümliche hinzu: Auslassungen 126, 5. 132, 16 usw. - Fehler oder Änderungen: 7, 21. 8, 8. 13, 10/11. 95, 1. 125, 2. 149, 1. 153, 6. 183, 11 usw. Daß anderseits gewisse Lesarten der Überlieferung $\mathbf{v}(\mathrm{z}$. B. 164, 20/21) auf sehr alte Varianten zurückgehen, zeige ich unten (S. LI), wo ich von der Tripartita spreche.

$\mathrm{V}$ ist der Archetypus des Venetus 337 und durch diesen des Codex Pini, die beide auf die frühern Ausgaben eingewirkt haben. Aufmerksam zu machen ist auf die Tatsache, daß V sich innerhalb der Gruppe $\mathrm{v}$ oft durch ihm eigentümliche Änderungen auszeichnet: 10, 4/5, eine seit Christophorson und Scaliger bekannte Änderung; 12, 5 u. 18 Änderungen im Anschluß an den Bibeltext usw.

R. Über eine dritte, nur als Bruchstück erhaltene Handschrift der Gruppe $\mathbf{v}$ will ich mich nicht ausführlicher verbreiten. Die erste Hand und an andern Stellen eine zweite haben darin oft die Fehler von $\nabla$ nach Lesarten von $L$ gebessert. Ich habe im Apparat diese Correcturen, die kein neues Element bringen, nicht berücksichtigt. Interesse haben sie nur, indem sie zeigen, daß die Handschriften fortwährend durcheinander beeinflußt sind und daß es daher nicht Wunder nehmen darf, wenn ihre Gruppierung auf Schwierigkeiten stößt.

\section{Der Wert von $B$ und $B^{4}$}

Vom dritten Buch ab erfreuen wir uns in einer sich ziemlich gleich bleibenden Weise der Unterstützung von B, zu der bald noch das Zeugnis von $\mathrm{V}^{2}$ oder $\mathrm{V}$ und in geringerem Grade (von S. $249 \mathrm{ab}$ ) das von F hinzutritt (s. unten S.XXXIX f). Die minderwertige Überlieferung wird von da an der Reihe nach durch $\mathrm{AL}(\mathrm{y})$ und $\mathrm{F}$ oder allein durch y dargestellt.

B. Die vollständige Beschreibung der Handschrift B habe ich bisher aufgeschoben und oben (S. XXXIIf) nur auf einige Übereinstimmungen hingewiesen, die seine Verwandtschaft mit $\mathrm{A}$ im ersten Teile dartun.

Hinsichtlich der Orthographie ist B eine Handschrift, die man nicht zur Richtschnur nehmen kann; sie ist voll von falschen Accenten, Itacismen 
und andern fehlerhaften Schreibungen, mit denen ich den kritischen Apparat nicht belastet habe. B schreibt z. B. immer öoıo und nicht

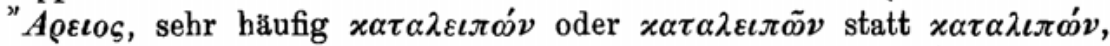

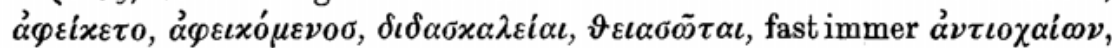

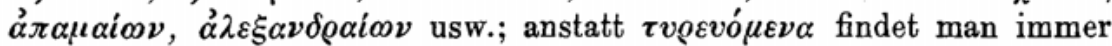
die Form $\tau v \delta \varepsilon v o ́ n \varepsilon v \alpha$. Es kommt vor, daß B die Umwandlung in die

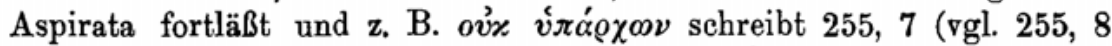

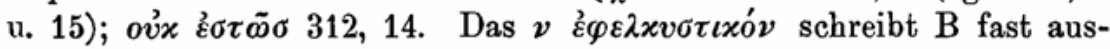
nahmslos, mag es im Satzeinschnitt sein oder nicht, vor Vocal oder Consonant.

Auslassungen sind bei ihm ziemlich zahlreich, z. B. 98, 7. 105, 17/18. $160,10 / 11$. 168, 14. 201, 17. 220, 7. 254, 10. 259, 1. 268,11. 301, 12/13. $318,14 / 15.341,20$.

Diese Auslassungen sind aber immer unbeabsichtigt. Ebenso bezeugen grobe Fehler und unsinnige Lesarten, daß $\mathrm{B}$ im allgemeinen von gelehrten Besserungen frei geblieben ist. Ich nenne als Bei-

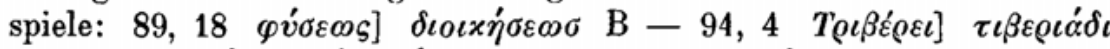

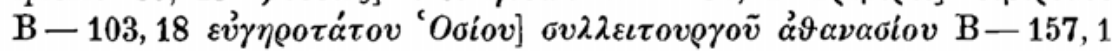

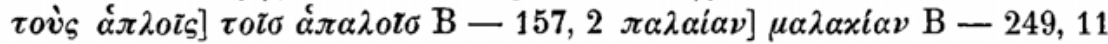

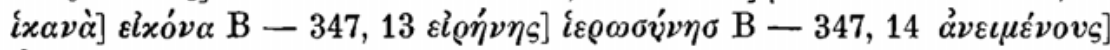

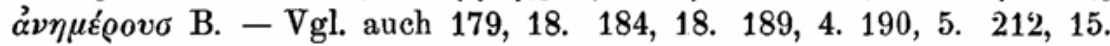
214,20 . 227, 16. $239,3,13$ u. 20. 249, 21. 319, 11. 344, 1 usw.

Die Häufung der Fehler an manchen Stellen scheint auf den schlechten Zustand des Archetypus zurückzugehen; vgl. z. B. die Urkunde II 2 (S. 94, $10 \mathrm{ff}$ ), wo B durchweg recht fehlerbaft ist.

Änderungen und Zusätze sind selten und wenig erheblich; sie lassen sich gewöhnlich durch Vergleichung mit Cass. oder mit der Parallelüberlieferung erkennen. Einige Beispiele bieten: 154, 5. 182, 16. 190, 2. 201,3 u. 4 . $202,23.206,4$ u. 13. 210, 3. 242, 23. 282, 6. 314, 5.

Unter den Lesarten von B, die ich verworfen habe, ist als besonders

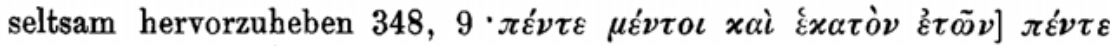

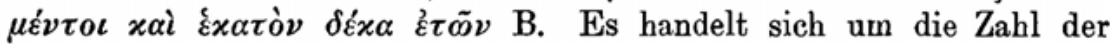
Jahre, 105 oder 115, welche die Geschichte des Theodoret umfaßt. Den Grund, der hier zur Verwerfung des Zeugnisses von B führen muß, habe ich im kritischen Apparat angegeben.

B und Cassiodor. Das Alter der Überlieferung von B und die Tatsache, daß B viele Entstellungen der Überlieferung von $\mathrm{z}$ oder $\mathrm{yF}$ vermeidet, lassen sich am besten beweisen durch die enge Verwandtschaft seines Textes mit dem der Handschrift, deren sich Cassiodor bedient hat.

$B$ und Cass. haben gemeinsame Auslassungen, die zu zahlreich und 
zu charakteristisch sind, um zufällig zu sein: 228, 17/18. 238, 3. 259, 10. 260, 9. 333, 3 .

B und Cass. stehen allein mit fehlerhaften oder zweifelhaften Lesarten, die ich geglaubt habe verwerfen zu müssen: 154, $\left.8 \dot{\varepsilon} \times x \times \lambda \eta \sigma \iota \sigma \sigma \tau \iota x \tilde{\eta}_{S}\right]$

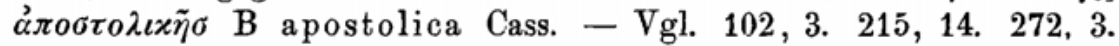

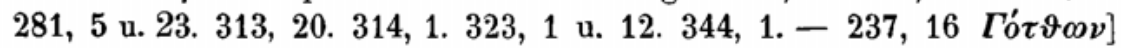
$x \varepsilon \lambda \tau \tilde{\omega \nu}$ B Celtis Cass. - 330, $\left.9 \sum x v \vartheta \imath x \dot{\partial} \nu\right] x \varepsilon \lambda \tau \iota x \dot{\nu} \nu$ B = Cass. 331, $12 \Sigma x \dot{\vartheta} \eta \zeta]$ ] $x^{\prime} \lambda \tau \iota o \sigma$ B Celticus Cass. In den beiden letzten Fällen zeigt die Parallelüberlieferung (s. krit. App.), daß die Variante von B Cass. in den ältesten Handschriften sehr verbreitet war.

Ebenso sind wegen ihres Alters besonders lehrreich die Fehler, in denen $B$ und Cass. mit andern Handschriften übereinstimmen: 104, 8

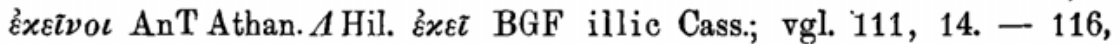

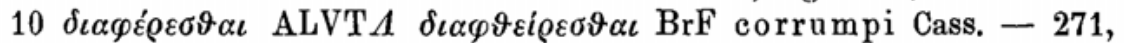
$\left.11 \varrho \varrho^{\prime} \gamma \mu \alpha \tau \alpha\right] \varrho \eta^{\prime} \mu \alpha \tau \alpha \mathrm{BV}{ }^{2} \mathrm{FN}$ verba Cass.

Außerordentlich zahlreich sind die Fälle, wo B und Cass. die richtige Lesart bewahrt haben. Schlägt man den kritischen Apparat für die hier notierten Stellen nach, so sieht man die Lesarten beider immer von der Parallelüberlieferung bestätigt: 101, 9. 183, 15. 221, 14. 232, 15. 272, 8. Besonders in der ganzen Urkunde V 11 (S. 297, $12 \mathrm{ff})$ haben allein B und Cass. unter fortwährender Bestätigung von seiten des Lateinischen sich von der Überarbeitung freigehalten, die alle übrigen Handschriften mehr oder weniger betroffen hat. Vgl. noch 309,15 $\tau \tilde{\omega} \nu . . \lambda \dot{o}^{\prime} \gamma \omega \nu .312,2$ usw.

Solchen Beispielen müßte man natürlich noch die zahlreichen Fälle hinzufügen, wo, wie wir sehen werden, gemeinsame richtige Lesarten von $\mathrm{B}$ und $\mathrm{V}^{2}$ durch Cass. bestätigt werden (S. XXXIX).

Danach ist es verständlich, daß ich dazu gekommen bin Lesarten aufzunehmen, die oft nur B und Cass. angehören; z. B. 191, 10. 221, 9. 231, 7. 233, 23. 337, 18. 343, 20. 345, 1/2. 346, 18/19. 347, 20.

Anderwärts ist es die Parallelüberlieferung, die, wenn Cass. fehit, den Wert der Lesart von B bezeugt; z. B. 303, 15/16 xà oủdéva

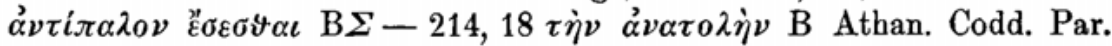
Bas. $\alpha \dot{\alpha} \alpha \tau \tau o \lambda \grave{\eta} v$. Tim. $\dot{\alpha} \nu \alpha \tau o \lambda \dot{\alpha} \sigma \mathrm{V}^{2} \mathrm{ryF}$. Unter diesen Umständen ist es nicht verwunderlich, daß B oft allein die Lesart geliefert hat, die den Vorzug zu verdienen schien; z. B. 231, 15/16. 239, 7. 251, 13. 304, 4. $324,15.325,6$. 326, 6 .

Die Interpolation in B. Seltsam ist es, daß allein die für gewöhnlich so zuverlässige Handschrift $\mathrm{B}$ eine lange Einschiebung (S. $307 \mathrm{f}$ Anmerkung) enthält, welche die Ausgabe von Gaisford in den Text aufgenommen hat. Es handelt sich um einen Brief des Bischofs 
von Thessalonich Acholius, worin er Ambrosius das im Auftrag des Theodosius erfolgte Blutbad in seiner Stadt mitgeteilt bätte. ZurZeit dieses Ereignisses (390) war indeß Acholius seit mehreren Jahren tot und Bischof von Thessalonich war Anysius. Dieser Beweisgrund würde jedoch nicht entscheidend sein, denn Theodoret verfährt oft nach seinem Belieben mit der Chronologie, und zwar besonders in der Angelegenheit des Blutbades von Thessalonich und des Aufruhrs von Antiochien. Aber hier sind die alten Abschriften im Überfluß vorhanden - syrische Übersetzung, Symeon Vita Ambrosii, Georgius Monachus, Cedrenus, Cassiodor und alle stimmen darin überein, daß sie den angeblichen Brief des Acholius nicht kennen. Ebenso wenig kennen ihn Berichte, die aus einer andern Überlieferung schöpfen.

Man braucht überdies nur zu lesen, in welcher Weise der Brief eingeführt wird, um sich zu überzeugen, daß der Übergang ungeschickt ist, den Satz unterbricht und ihn unrichtig macht. Das Stück selbst ist ein geschraubtes Klagelied, aus einer wunderlichen Folge von Anspielungen auf die heilige Schrift und schwälstigen Bildern zusammengesetzt, so daß Ambrosius bei seiner Lektüre sehr in Verlegenheit gewesen wäre zu erraten, um was es sich handelte. Das einzige etwas

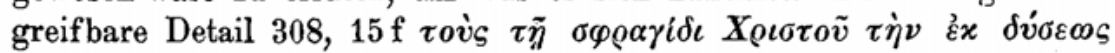

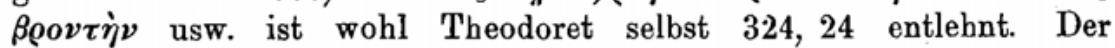
Schreiber von B hat das Stück so, wie er es in seiner Quelle fand, treu copiert. Entstanden ist es wohl als die Stilübung eines Priesters, der ein früberer Besitzer in seinem Exemplar des Theodoret diese Stelle zugewiesen hatte.

$\mathrm{B}^{4}$. Die Seiten $282,21-297,11$ sind in $\mathrm{B}$ von einer vierten Hand ergänzt worden. Daß B ${ }^{4}$ schadhaft gewordene Seiten aus B selbst neu abgeschrieben habe, halte ich für ausgeschlossen. Ebensowenig wie $\mathrm{B}^{2}$ und $\mathrm{B}^{3}$ zeigt $\mathrm{B}^{4}$ eine Spur der so charakteristischen orthographischen Besonderheiten von B. Dennoch gibt $\mathrm{B}^{4}$ eine gute Handschrift wieder, die VF und der des Nicephorus ziemlich nahe steht. Weiter unten wird sich zeigen, daß die Handschriften der Sippe $\mathbf{v}$ im fünften Buch das Eindringen der Recension $\mathrm{z}$ im allgemeinen vermeiden.

Obwohl sich $\mathrm{B}^{4} \mathrm{im}$ allgemeinen $\mathrm{zu} \mathrm{V}^{2} \mathrm{~F}$ in ihren guten Lesarten stellt (283, 14. 290, 16 usw.), begegnen charakteristische fehlerhafte

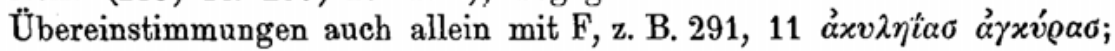

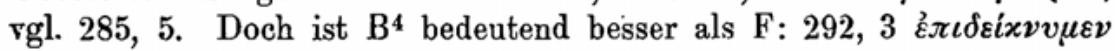
$B^{4} V W$ Cass. $>$ Fry; vgl. 294, 22. 295, 8 u. 15. 297, 2.

$\mathrm{B}^{4}$ verrät in allen diesen Fällen und in einigen andern noch bezeichnenderen $(290,21.294,12)$ besondere Verwandtschaft mit der Überlieferung des Nicephorus Callistus. 


\section{Der Wert von $\mathrm{V}^{2}$ oder $\mathrm{V}$ und (von S. 249 ab) F}

Von 187, 15 ab ändert sich die Handschrift $\mathrm{V}$ hinsichtlich der Schrift $\left(\mathrm{V}^{2}\right)$ und auch des Wertes. $\mathrm{V}^{2}$ hört auf, die Fehler der Recension $\mathrm{z}$ (Fy oder $\mathrm{rFy}$ ) zu bieten, und wird mit B der Vertreter einer bessern Überlieferung. Und auch wo im fünften Buch die erste Hand $(V)$ wiedereinsetzt, bewahrt die Handschrift im allgemeinen ihren neuen Wert.

Gemeinsame Fehler zeigen die Verwandtscbaft der Überlieferung von $\mathrm{V}^{2}$ mit B: 203, 24. 214, 14. 217, 8. - 220, 12 ह่ $\pi \iota \pi \varepsilon \rho \iota \beta \alpha \lambda \lambda o v ́ \sigma \alpha \sigma$

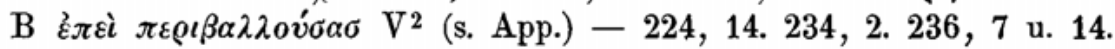
$244,20.256,22 / 23.258,12.272,13$ u. 14 usw.

Anderseits stimmt $\mathrm{V}^{2}$ (oder $\mathrm{V}$ ) in vielen Fällen mit $\mathrm{B}$ in richtigen Lesarten überein gegen die Gesamtheit der andern Handschriften ryF. Ihre Lesarten findet man im kritischen Apparat bei allen Stellen, auf die ich hier verweise, im allgemeinen von Cass. oder von der Parallelüberlieferung bestätigt: $201,10.202,18$ u. 24. 205, 7. 212, 4. 217, 1 u. $21.224,2 / 3.234,4$ u. $11 / 12$. $260,21.270,16.273,10.316,24$ usw. Siehe auch 224, 1, wo allein BV ${ }^{2}$ Cass.Nic. ein theologisches Einschiebsel der andern Handschriften vermeiden, und 266, 19, wo wieder sie allein nicht die Besserung óøiov statt i is ovv einführen.

$\mathrm{V}^{2}$ (oder V) hat, obwohl der Codex mit vieler Sorgfalt geschrieben ist, ihm eigentümliche Fehler in ziemlich großer Zahl. Auslassungen liegen vor: $197,9.213,12$ u. 18. $227,2.313,9.319,5 / 6$ usw.

Unter den sonstigen Fehlern sei auf eine Anzahl verwiesen, deren gemeinsames Kennzeichen ist, daß sie das Wort des Textes willkürlich durch eine andere Form oder durch ein Synonymum, zuweilen sogar durch einen Ausdruck anderen Sinnes ersetzen. $V^{2}(V)$ ist tatsächlich weit weniger frei von Änderungen als B, und da manche dieser Lesarten, für sich genommen, bestechend scheinen könnten, empfiehlt es sich, einige Fälle zusammenzustellen: 192, 1. 199, 5. 203, 8. 204, 16. 205, 17. 206, 4. 214, 6. 216,21 u. 24. 224 , 5. 231, 11. 235, 20. $247,1$. 252 , 1. 254 , 15. 258,22 . 261,6 . 262 , 5/6. $266,11.269$, 3. 271, 1. 283 , 20. 286 , 3. 319 , 4. 326,16 . 329 , 19. 332 , 10/11. 348 , 1.

Trotz dieser Einschränkungen bleibt $V^{2}$ neben $B$ eine sehr nützliche Handschrift, die wertvolle Lesarten geliefert hat: 237, 7 hat sie allein

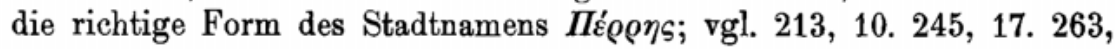
19. 265,19 (s. krit. App.). 326, 5. - 272, 3-5 habe ich nur ein Wort in $\mathrm{V}^{2}$ zu verbessern gehabt ( $\left(\varepsilon \sigma \tau \iota v\right.$ in $\left.\eta^{\prime} \sigma \tau \eta \nu\right)$, um den ursprünglichen Text vollkommen wiederherzustellen, von dem allein $\mathrm{V}^{2}$ die beiden andern Duale unversehrt bewahrt hatte.

Ein besondres Interesse ist mit solchen Lesarten von. $\mathrm{V}^{2}$ oder $\mathrm{V}$ 
verknüpft, die als Varianten der Quelle anzusehen statthaft ist, wie ich

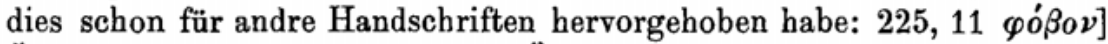

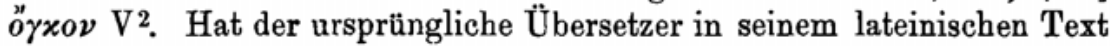
zwischen timorem und tumorem geschwankt? Als alte Varianten sind wohl auch einige sehr merkwürdige Übereinstimmungen zwischen

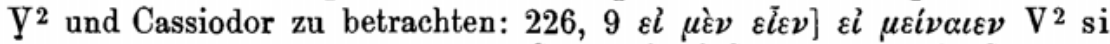

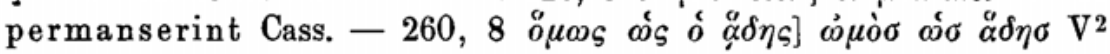

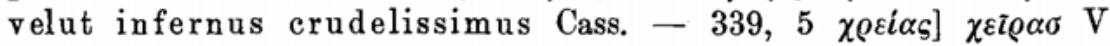
manus Cass.

F. Ungefähr von S. 249 ab haben die charakteristischen Übereinstimmungen von y (AL) und F, auf die ich demnächst zu sprechen komme, die Tendenz aufzuhören, und der Codex $\mathrm{F}$ bietet gewöhnlich Lesarten, die ihn $\mathrm{V}^{2}$ oder $\mathrm{V}$ nähern. So stimmt er oft mit $\mathrm{BV}^{2}$ überein und vermeidet Febler von ry: 250,18 . 251, 15. 255, 7. 263, 17/18. 269, 6. 279, 9. 299, 18 ff. $301,5.310,1.323,21$ usw.

Das Auseinandergehen von $\mathrm{V}^{2}(\mathrm{~V}), \mathrm{F}$ (und auch von W, s. S. XLV) in diesem Teil dürfte sich - man vergleiche den Fall von $n$ und $s$ für die Gruppe $\mathrm{r}$ im ersten Buch, S. XXVII - durch doppelte Lesarten in dem Archetypus $\mathbf{v}$ erklären. $\mathrm{F}$ hat sorgfältiger als $\mathrm{V}^{2}(\mathrm{~V})$ daran festgehalten, die Varianten der Recension z zu berücksichtigen (z. B. 276, 23. 277, 6. 280,6 usw.). Daß trotzdem $V^{2}(V)$ und $F$ immer noch von derselben Vorlage abstammen, wird durch eine Anzahl gemeinsamer Fehler erwiesen: $256,13.283,15.298,5$ u. $15.299,2$ u. 8. 301,13 und 23 ff. $303,4.317,3 / 4.326,8$ u. 11. 327,7 usw.

\section{Die Gruppe $\mathrm{AL}(\mathrm{y})$ und $\mathrm{yF}$}

Es wurde oben behauptet, daß A vom dritten Buch ab seine vortreffliche Überlieferung vom Anfang völlig aufgibt und sich in eine Zwillingshandschrift von $\mathrm{L}$ verwandelt.

Diese scheinbar verwickelte Sachlage läßt sich jedoch auf ganz ungezwungene Art erklären. Man braucht nur den identischen Inbalt der beiden Handschriften und die verschiedene Ordnung zu beachten, in der die Werke sich folgen.

L enthält: 1. Theodoreti Graecarum affectionum curatio. 2. Eiusdem ecclesiastica historia. 3. Eiusdem de providentia orationes decem. A enthält dieselben Werke mit dem Unterschied in der Reihenfolge, daß die Kirchengeschichte an erster Stelle steht, dann de providentia orationes und zum Schluß Graecarum affectionum curatio.

W as hat sich also zugetragen? Der Schreiber von A hatte angefangen die Kirchengeschichte nach einer alten Handschrift von der Art der guten 
Quelle von $\mathrm{n}$ zu copieren. Im Laufe seiner Arbeit entdeckt er eine schöne Handschrift einer Recension, die in gelehrter d. b. schädlicher Weise corrigiert worden war; dies ist die Quelle von L. Durch unzählige Rasuren macht nun A den ersten Teil seines Textes mit dieser neuen Handschrift gleichlautend und vollendet etwa vom dritten Buche ab nach ihr allein seine Abschrift. Er fährt dann fort, aus ihr die zehn Reden über die Vorsehung zu copieren, die in der Quelle unmittelbar folgten, entscheidet sich schließlich, sie ganz wiederzugeben, und fügt so Graecarum affectionum curatio, die an der Spitze stand, hinten an. In seiner vortrefflichen kritischen Ausgabe des letzteren Werkes hat Raeder, woran erinnert sein möge, nachgewiesen, daß die beiden Handschriften L und A(bei Raeder B genannt) sehr eng verwandt sind.

Die Gruppe y bietet natürlich weiter die Merkmale, die ich für $\mathrm{L}$ allein hervorgehoben habe (S. XXXV). Sie stimmt gelegentlich in richtigen Lesarten mit den besten Zeugen gegen $r$ und $\mathrm{v}$ überein, $\mathrm{z} . \mathrm{B}$. 183,8 u. 11. 186, 19 usw. $-196,14$ hat y allein die Lesart $\vartheta \alpha v \mu \alpha_{-}-$ $\zeta o v$ bewahrt, die mir die sicherste schien; vgl. auch 216, 18.

Es liegt mir noch ob, ein Wort über die Lesarten von $\mathrm{A}^{\mathrm{c}}$ zu sagen; sie sind in diesem zweiten Teil weit seltener. Es kommt wenig darauf an zu bestimmen, ob diese Varianten, wie ich geneigt bin zu glauben, in der Quelle y selbst sich fanden oder ob sie $\mathrm{A}^{\mathrm{c}}$ nunmehr einer andern Handschrift entlehnt. Die Lesarten von $\mathrm{A}^{\mathrm{c}}$ sind im allgemeinen gleichlautend mit F: vgl. 185, 16. 186, 7. 193, 6. 196, 6, und zuweilen selbst allein mit F: 223, $1 \delta \alpha i \lambda \dot{\alpha} \mu \pi \omega \nu \sigma \sigma \mathrm{FA}^{\mathrm{e}}$ und Nic.; vgl. 242, 3. 249, 7. Die Correcturen sind übrigens mechanisch und ohne Kritik gemacht: 196, 8 u. 15. 299, 2. Hinzuzufügen ist, daß die Quelle für $\mathbf{A}^{c}$ sicher nicht $\mathrm{F}$ selbst ist, sondern unter Umständen besser als $\mathrm{F}$ ist, ein Verhältnis, das wir auch zwischen $\mathrm{F}$ und der Handschrift des Nicephorus

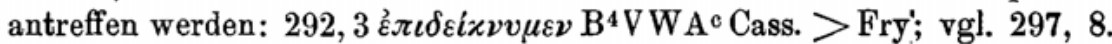

Die Gruppe yF. Ebenso wie in den beiden ersten Büchern L oft mit $\mathrm{FV}(\mathrm{v})$ eine Gruppe bildete, schließt $\mathrm{AL}(\mathrm{y})$ begreiflicherweise sich weiter an $\mathrm{F}$ an, wenn $\mathrm{F}$ nach dem Auftreten von $\mathrm{V}^{2}$ allein die Fehler jener Sippe darstellt.

Auslassungen in $\mathrm{yF}: 204,3$ déov $>\mathrm{yF}-230,16$ xai $\pi \lambda \eta \sigma i o v$ - $x \varepsilon \lambda \varepsilon v \sigma \alpha \alpha_{\varsigma}>\mathrm{yF}$; vgl. 233, 21. 234, 11/12 u. 23. 235, 25. $245,4$.

Andere fehlerhafte Übereinstimmungen: 204, $13 \dot{\alpha} \pi \varepsilon \iota \lambda \tilde{\omega} \nu]$ $x \varepsilon \alpha v \nu \tilde{\omega} \nu$ $\left.\mathrm{yF}-211,10 \varepsilon v^{\prime} \sigma \varepsilon \beta \varepsilon \sigma \epsilon\right] \quad \varepsilon \dot{v} \sigma \varepsilon \beta \varepsilon i \alpha \sigma \mathrm{yF}$; vgl. 216, 24. 217, 17. 238, 6 usw. Zusätze oder Änderungen: $193,22+o$ ov $\tau \omega \sigma \mathrm{yF}-204,4+\delta \delta \dot{\varepsilon}$

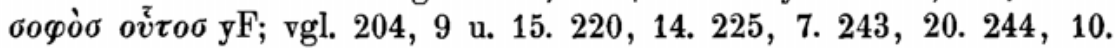

Von S. 249 ab scheidet $F$, wie wir gesehen haben, im allgemeinen aus dieser Gruppe aus, um sich $\mathrm{V}$ wieder zu nähern. 